\title{
DIFFERENT SIMILARITIES
}

\begin{abstract}
Miloš S. Kuriliéd
Abstract

We establish the hierarchy among twelve equivalence relations (similarities) on the class of relational structures: the equality, the isomorphism, the equimorphism, the full relation, four similarities of structures induced by similarities of their self-embedding monoids and intersections of these equivalence relations. In particular, fixing a language $L$ and a cardinal $\kappa$, we consider the interplay between the restrictions of these similarities to the class $\operatorname{Mod}_{L}(\kappa)$ of all $L$-structures of size $\kappa$. It turns out that, concerning the number of different similarities and the shape of the corresponding Hasse diagram, the class of all structures naturally splits into three parts: finite structures, infinite structures of unary languages, and infinite structures of non-unary languages (where all these similarities are different). 2010 Mathematics Subject Classification: 03C07, 20M20, 06A06, 03 E40. Keywords: relational structure, isomorphic substructure, partial order, selfembedding monoid, isomorphism, equimorphism, forcing-equivalence.
\end{abstract}

\section{Introduction}

If $\mathbb{X}$ is a relational structure, $\operatorname{Emb}(\mathbb{X})$ the monoid of its self-embeddings and $\mathbb{P}(\mathbb{X})=\{f[X]: f \in \operatorname{Emb}(\mathbb{X})\}$ the set of copies of $\mathbb{X}$ inside $\mathbb{X}$, then the poset $\langle\mathbb{P}(\mathbb{X}), \subset\rangle$ (isomorphic to the inverse of the right Green's order on $\operatorname{Emb}(\mathbb{X})$ ) contains a certain information about $\mathbb{X}$ and the equality $\mathbb{P}(\mathbb{X})=\mathbb{P}(\mathbb{Y})$ defines an equivalence relation on the class of all relational structures. Writing $\mathbb{P}(\mathbb{X})$ instead of $\langle\mathbb{P}(\mathbb{X}), \subset\rangle$, some coarser classifications of structures are obtained if the equality is replaced by the following weaker conditions: $\mathbb{P}(\mathbb{X}) \cong \mathbb{P}(\mathbb{Y})$ (implied by $\operatorname{Emb}(\mathbb{X}) \cong \operatorname{Emb}(\mathbb{Y})$ ), sq $\mathbb{P}(\mathbb{X}) \cong \mathrm{sq} \mathbb{P}(\mathbb{Y})$ (where sq $\mathbb{P}$ denotes the separative quotient of a poset $\mathbb{P})$, and $\mathbb{P}(\mathbb{X}) \equiv \mathbb{P}(\mathbb{Y})$ (the forcing equivalence of posets of copies). Concerning the last (and the coarsest non-trivial) similarity relation we note that the forcing related properties of posets of copies was investigated for countable structures in general in [6], for equivalence relations and similar structures in [7], for ordinals in [8], for scattered and non-scattered linear orders in [9] and [11], and for several ultrahomogeneous structures in [10],[11],[12], and [13].

In this paper we investigate the interplay between the four similarity relations mentioned above and the similarities defined by the conditions $\mathbb{X}=\mathbb{Y}, \mathbb{X} \cong \mathbb{Y}$, and $\mathbb{X} \rightleftarrows \mathbb{Y}$ (equimorphism, bi-embeddability).

\footnotetext{
${ }^{1}$ Department of Mathematics and Informatics, University of Novi Sad, Trg Dositeja Obradovića 4, 21000 Novi Sad, Serbia. e-mail: milos@dmi.uns.ac.rs
} 
In Section 2 we establish the hierarchy displayed in Figure 1, which, more precisely, contains the implications between the similarities on the class of pairs $\langle\mathbb{X}, L\rangle$, where $L$ is a language and $\mathbb{X}$ an $L$-structure. (The language must be included in the game because, otherwise, since the structure $\mathbb{X}=\langle\omega,\langle\emptyset\rangle\rangle$ can be regarded as an $L$-structure for each language $L$ of size 1 , it is not clear what $\mathbb{X} \cong \mathbb{Y}$ means). So, the conditions displayed in the diagram define when the pairs $\left\langle\mathbb{X}, L_{1}\right\rangle$ and $\left\langle\mathbb{Y}, L_{2}\right\rangle$ are similar (clearly, the equality $L_{1}=L_{2}$ follows from $\mathbb{X} \cong \mathbb{Y}$ and $\mathbb{X} \rightleftarrows \mathbb{Y}$ and we omit it). Thus, for example, line $n$ denotes the statement that equimorphic structures have forcing-equivalent posets of copies.

In Section 3 we fix a language $L$ and a set $X$ and restrict our analysis to the class $\operatorname{Mod}_{L}(X)$ of $L$-structures with the domain $X$. It turns out that for a nonunary language $L$ and infinite set $X$ in the diagram from Figure 1 restricted to the class $\operatorname{Mod}_{L}(\kappa)$ all the implications $a-o$ are proper and there are no new implications (except the ones following from transitivity). On the other hand, for finite structures or unary languages the diagram collapses significantly.

A few words on notation. Let $L=\left\langle R_{i}: i \in I\right\rangle$ be a relational language, where $\operatorname{ar}_{L}\left(R_{i}\right)=n_{i} \in \mathbb{N}, i \in I$ and let $X$ be a non empty set. If $\mathbb{X}=\left\langle X,\left\langle\rho_{i}: i \in I\right\rangle\right\rangle$ is an $L$-structure and $\emptyset \neq A \subset X$, then $\left\langle A,\left\langle\rho_{i} \uparrow A: i \in I\right\rangle\right\rangle$ is a substructure of $\mathbb{X}$, where $\rho_{i}\left\lceil A=\rho_{i} \cap A^{n_{i}}\right.$, for $i \in I$. If $\mathbb{Y}=\left\langle Y,\left\langle\sigma_{i}: i \in I\right\rangle\right\rangle$ is an $L$-structure too, a mapping $f: X \rightarrow Y$ is an embedding (we write $f: \mathbb{X} \hookrightarrow \mathbb{Y}$ ) iff $f$ is an injection and for all $i \in I$ and $x_{1}, \ldots x_{n_{i}} \in X$ we have $\left\langle x_{1}, \ldots, x_{n_{i}}\right\rangle \in \rho_{i} \Leftrightarrow$ $\left\langle f\left(x_{1}\right), \ldots, f\left(x_{n_{i}}\right)\right\rangle \in \sigma_{i}$. Let $\operatorname{Emb}(\mathbb{X}, \mathbb{Y})$ denote the set of such embeddings and $\mathbb{P}(\mathbb{X}, \mathbb{Y})=\left\{B \subset Y:\left\langle B,\left\langle\sigma_{i}\lceil B: i \in I\rangle\right\rangle \cong \mathbb{X}\right\}=\{f[X]: f \in \operatorname{Emb}(\mathbb{X}, \mathbb{Y})\}\right.$. In particular, $\operatorname{Emb}(\mathbb{X}):=\operatorname{Emb}(\mathbb{X}, \mathbb{X})$ and $\mathbb{P}(\mathbb{X}):=\{f[X]: f \in \operatorname{Emb}(\mathbb{X})\}=$ $\left\{A \subset X:\left\langle A,\left\langle\rho_{i} \mid A: i \in I\right\rangle\right\rangle \cong \mathbb{X}\right\}$. If $f \in \operatorname{Emb}(\mathbb{X}, \mathbb{Y})$ is a surjection, it is an isomorphism, we write $f \in \operatorname{Iso}(\mathbb{X}, \mathbb{Y})$, and the structures $\mathbb{X}$ and $\mathbb{Y}$ are isomorphic, in notation $\mathbb{X} \cong \mathbb{Y}$. If, in particular, $\mathbb{Y}=\mathbb{X}$, then $f$ is called an automorphism of the structure $\mathbb{X}$ and Aut $(\mathbb{X})$ denotes the set of all such mappings. Structures $\mathbb{X}$ and $\mathbb{Y}$ are called equimorphic, in notation $\mathbb{X} \rightleftarrows \mathbb{Y}$, iff $\mathbb{X} \hookrightarrow \mathbb{Y}$ and $\mathbb{Y} \hookrightarrow \mathbb{X}$.

The right Green's pre-order $\preceq^{R}$ on the monoid $\left\langle\operatorname{Emb} \mathbb{X}, \circ, \mathrm{id}_{X}\right\rangle$ is defined by:

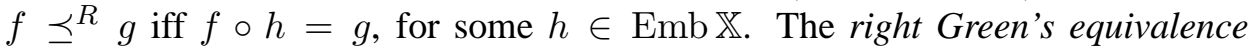
relation $\approx^{R}$ on Emb $\mathbb{X}$, given by: $f \approx^{R} g$ iff $f \preceq^{R} g$ and $g \preceq^{R} f$, determines the antisymmetric quotient $\left\langle\operatorname{Emb} \mathbb{X} / \approx^{R}, \preceq^{R}\right\rangle$, the right Green's order. It is easy to check that $\left\langle\operatorname{Emb} \mathbb{X} / \approx^{R}, \preceq^{R}\right\rangle \cong\langle\mathbb{P}(\mathbb{X}), \supset\rangle$ so the results of this paper can be regarded as statements about transformation semigroups.

A partial order $\mathbb{P}=\langle P, \leq\rangle$ is called separative iff for each $p, q \in P$ satisfying $p \not \leq q$ there is $r \in P$ such that $r \leq p$ and $r \perp q$. The separative modification of $\mathbb{P}$ is the pre-order sm $\mathbb{P}=\left\langle P, \leq^{*}\right\rangle$, where $p \leq^{*} q$ iff $\forall r \leq p \exists s \leq r s \leq q$. The separative quotient of $\mathbb{P}$ is the separative partial order sq $\mathbb{P}=\left\langle P /=^{*}, \unlhd\right\rangle$, where $p={ }^{*} q \Leftrightarrow p \leq^{*} q \wedge q \leq^{*} p$ and $[p] \unlhd[q] \Leftrightarrow p \leq^{*} q$. If $\mathbb{P}$ is a separative partial 


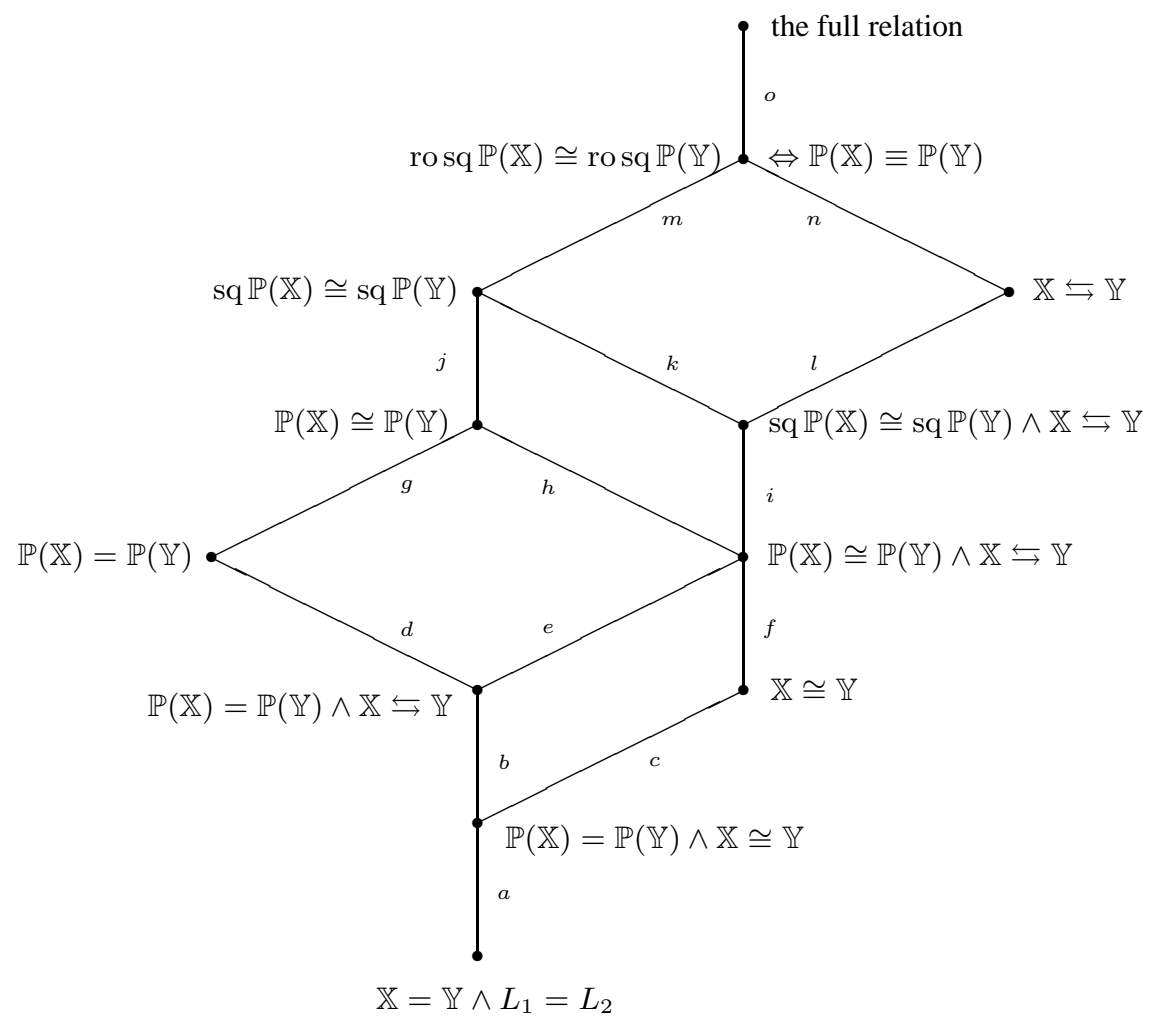

Figure 1: The hierarchy of similarities between relational structures

order, by ro $\mathbb{P}$ we will denote the Boolean completion of $\mathbb{P}$. For a pre-order $\mathbb{P}$ let $\operatorname{ge}(\mathbb{P})=\left\{V_{\mathbb{P}}[G]: G\right.$ is a $\mathbb{P}$-generic filter over $\left.V\right\}$. Two pre-orders $\mathbb{P}$ and $\mathbb{Q}$ are said to be forcing equivalent, in notation $\mathbb{P} \equiv \mathbb{Q}$, iff ge $(\mathbb{P})=\operatorname{ge}(\mathbb{Q})$.

Fact 1.1 Let $\mathbb{P}, \mathbb{Q}$ and $\mathbb{P}_{i}, i \in I$, be partial orderings. Then

(a) $\mathbb{P} \cong \mathbb{Q} \Rightarrow \operatorname{sm} \mathbb{P} \cong \operatorname{sm} \mathbb{Q} \Rightarrow \mathrm{sq} \mathbb{P} \cong \mathrm{sq} \mathbb{Q} \Rightarrow \operatorname{rosq} \mathbb{P} \cong \operatorname{rosq} \mathbb{Q} \Rightarrow \mathbb{P} \equiv \mathbb{Q} ;$

(b) $\mathbb{P} \equiv \operatorname{sm} \mathbb{P} \equiv \operatorname{sq} \mathbb{P} \equiv(\operatorname{rosq} \mathbb{P})^{+}$;

(c) $\operatorname{sq}\left(\prod_{i \in I} \mathbb{P}_{i}\right) \cong \prod_{i \in I} \mathrm{sq} \mathbb{P}_{i}$.

\section{Implications}

In this section we establish the implications $a-o$ from Figure 1 In Section 3 we will show that, regarding the class of all relational structures, there are no new implications in Figure 1 (except the ones which follow from the transitivity). First, the 
implications $a, b, c, d, e, g, h, k, l$, and $o$ are evident, while $i, j$ and $m$ follow from Fact 1.1 a). In the sequel we prove the equivalence rosq $\mathbb{P}(\mathbb{X}) \cong \operatorname{rosq} \mathbb{P}(\mathbb{Y}) \Leftrightarrow$ $\mathbb{P}(\mathbb{X}) \equiv \mathbb{P}(\mathbb{Y})$ and the implications $f$ and $n$ (see Theorems 2.7 and 2.10).

\subsection{Intermezzo: the homogeneity of Boolean completions}

Here we prove that the Boolean completion of the poset of copies of a relational structure is a homogeneous Boolean algebra. We recall that a partial order $\mathbb{P}=$ $\langle P, \leq\rangle$ is called homogeneous iff it has a largest element and $\mathbb{P} \cong p \downarrow$, for each $p \in P$ and that a Boolean algebra $\mathbb{B}$ is called a homogeneous Boolean algebra iff $\mathbb{B} \cong b \downarrow$, for each $b \in \mathbb{B}^{+}$. It is known that the Boolean completion of a separative homogeneous partial order $\mathbb{P}$ is a homogeneous Boolean algebra (see [4], p. 181) and, by Theorem 2.2 of [6], the posets of the form $\mathbb{P}(\mathbb{X})$ are homogeneous but it is easy to see that they are not separative in most of the cases. So, in order to prove that the Boolean completions rosq $\mathbb{P}(\mathbb{X})$ are homogeneous algebras, we show that in the theorem mentioned above the separativity of $\mathbb{P}$ can be omitted and that the assumption of homogeneity can be relaxed. Namely, defining a partial order $\mathbb{P}$ to be quasi homogeneous iff for each $p \in P$ there is a dense subset of $\mathbb{P}$ isomorphic to a dense subset of $p \downarrow$, we have the following generalization.

Theorem 2.1 The Boolean completion of a quasi homogeneous partial order $\mathbb{P}$ is a homogeneous Boolean algebra.

Proof. The statement is a consequence of the following two claims. Namely, if $\mathbb{P}$ is a quasi homogeneous partial ordering, then, by Claim 2.2, sq $\mathbb{P}$ is a separative quasi homogeneous partial order and, by Claim 2.3 , the algebra ro sq $\mathbb{P}$ is homogeneous.

Claim 2.2 The separative quotient of a quasi homogeneous partial order is quasi homogeneous.

Proof. Let $\mathbb{P}=\langle P \leq\rangle$ be a quasi homogeneous partial order, sq $\mathbb{P}=\left\langle P /=^{*}, \unlhd\right\rangle$ and $p \in P$. Let $D$ be a dense subset of $\mathbb{P}$ and $f:\langle D, \leq\rangle \rightarrow\langle p \downarrow, \leq\rangle$ an embedding such that $f[D]$ is a dense subset of $p \downarrow$. First we prove that

$$
\forall q, r \in D \quad\left(q \leq^{*} r \Leftrightarrow f(q) \leq^{*} f(r)\right) .
$$

Let $q, r \in D$. If $q \leq^{*} r$, then each $s \leq q$ is compatible with $r$ and we prove that each $u \leq f(q)$ is compatible with $f(r)$. If $u \leq f(q)$, then $u \leq p$ and, since $f[D]$ is dense in $p \downarrow$, there is $s \in D$ such that $f(s) \leq u$. Since $f$ is an embedding and $f(s) \leq f(q)$ we have $s \leq q$ and, since $q \leq^{*} r$, there is $t \leq s, r$, and, moreover there is $t^{\prime} \in D$ such that $t^{\prime} \leq t$ which implies $f\left(t^{\prime}\right) \leq f(s) \leq u$ and $f\left(t^{\prime}\right) \leq f(r)$. Thus $u \not \perp f(r)$. 
Assuming that $f(q) \leq^{*} f(r)$ and $s \leq q$ we show that $s \not \perp r$. If $s \leq q$ and $s^{\prime} \in D$, where $s^{\prime} \leq s$, then $f\left(s^{\prime}\right) \leq f(q)$ and, since $f(q) \leq^{*} f(r)$, there is $v \leq f\left(s^{\prime}\right), f(r) \leq p$. Since $f[D]$ is dense in $p \downarrow$, there is $t \in D$ such that $f(t) \leq v$. Since $f$ is an embedding we have $t \leq s^{\prime}, r$ and, hence, $s \not \perp r$. So (1D) is true.

It is evident that the set $\mathcal{D}:=\{[q]: q \in D\}$ is a dense suborder of the partial $\operatorname{order}\left\langle P /=^{*}, \unlhd\right\rangle$ and we prove that the mapping

$$
F:\langle\mathcal{D}, \unlhd\rangle \rightarrow\langle[p] \downarrow, \unlhd\rangle
$$

given by $F([q])=[f(q)]$, is an embedding. First, for $q, r \in D$ by (1) we have $[q]=[r]$ iff $q={ }^{*} r$ iff $q \leq^{*} r \wedge r \leq^{*} q$ iff $f(q) \leq^{*} f(r) \wedge f(r) \leq^{*} f(q)$ iff $f(q)={ }^{*} f(r)$ iff $[f(q)]=[f(r)]$ iff $F([q])=F([r])$ and, thus, $F$ is a well defined injection. Second, for $q \in D$ we have $f(q) \leq p$, which implies $f(q) \leq{ }^{*} p$ and, hence, $[f(q)] \unlhd[p]$, that is $F([q]) \in[p] \downarrow$. Thus $F[\mathcal{D}] \subset[p] \downarrow$. Finally, by (1), for $q, r \in D$ we have $[q] \unlhd[r]$ iff $q \leq^{*} r$ iff $f(q) \leq^{*} f(r)$ iff $[f(q)] \unlhd[f(r)]$ iff $F([q]) \unlhd F([r])$ and, thus, $F$ is a strong homomorphism.

Now we prove that $F[\mathcal{D}]$ is a dense set in the poset $\langle[p] \downarrow, \unlhd\rangle$. If $[q] \unlhd[p]$, then there is $s \leq p, q$ and, since $f[D]$ is dense in $p \downarrow$, there is $u \in D$ such that $f(u) \leq s$. Now, $f(u) \leq q$ implies $f(u) \leq * q$ thus $F([u])=[f(u)] \unlhd[q]$ and $F([u]) \in F[\mathcal{D}]$.

Thus the partial order sq $\mathbb{P}$ is quasi homogeneous indeed.

Claim 2.3 The Boolean completion of a separative quasi homogeneous partial ordering is a homogeneous complete Boolean algebra.

Proof. Let $\mathbb{P}=\langle P \leq\rangle$ be a separative quasi homogeneous partial order. First we show that

$$
\forall p \in P \quad \operatorname{ro} \mathbb{P} \cong \operatorname{ro}(p \downarrow) .
$$

If $p \in P$, then there is a dense subset $D$ of $\mathbb{P}$ and an embedding $f: D \hookrightarrow p \downarrow$ such that $f[D]$ is a dense subset of $p \downarrow$. Thus $D$ and $f[D]$ are isomorphic separative posets, which implies that ro $D \cong \operatorname{ro} f[D]$. In addition, $D$ is a dense suborder of the separative order $\mathbb{P}$, which, by the uniqueness of the Boolean completion, implies ro $\mathbb{P} \cong$ ro $D$ and, similarly, ro $f[D] \cong \operatorname{ro}(p \downarrow)$ and (2) is true.

Let $\mathbb{B}=\operatorname{ro} \mathbb{P}, b \in \mathbb{B}^{+}$and w.l.o.g. suppose that $\mathbb{P}$ is a dense suborder of $\mathbb{B}^{+}$. Then there is $p \in \mathbb{P}$ such that $p \leq_{\mathbb{B}} b$. Clearly the set $(p \downarrow)_{\mathbb{B}} \cap P=(p \downarrow)_{\mathbb{P}}$ is a dense suborder of the relative algebra $(p \downarrow)_{\mathbb{B}}$, which implies $(p \downarrow)_{\mathbb{B}} \cong \operatorname{ro}\left((p \downarrow)_{\mathbb{P}}\right)$ so, by (2), $(p \downarrow)_{\mathbb{B}} \cong$ ro $\mathbb{P} \cong \mathbb{B}$. It is well known that, if $\mathbb{B}$ is a $\sigma$-complete Boolean algebra, $a, b \in \mathbb{B}, a \leq b$ and $\mathbb{B} \cong a \downarrow$, then $\mathbb{B} \cong b \downarrow$ (see [4], p. 180). So we have $b \downarrow \cong \mathbb{B}$. 
Example 2.4 Clearly homogeneous partial orders are quasi homogeneous, but the converse is not true. Let $\mathbb{R}$ be the real line and

$$
\mathbb{P}=\langle\{(a, b]: a, b \in \mathbb{R} \wedge a<b\} \cup\{\mathbb{R}\}, \subset\rangle .
$$

Then for $p=(a, b]$ we have $p \downarrow \neq \mathbb{P}$, since the largest element of $\mathbb{P}$ is not the supremum of two smaller elements. Thus $\mathbb{P}$ is not a homogeneous partial order. On the other hand, if $f: \mathbb{R} \rightarrow(a, b)$ is an isomorphism, then it is easy to show that the mapping $F: P \rightarrow p \downarrow$ defined by $F(\mathbb{R})=p$ and $F((c, d])=(f(c), f(d)]$ is an embedding and that $F[P]$ is a dense subset of $p \downarrow$. Thus the partial order $\mathbb{P}$ is quasi homogeneous. We note that $\mathbb{P}$ is, in addition, separative.

Theorem 2.5 For each relational structure $\mathbb{X}$ the Boolean completion rosq $\mathbb{P}(\mathbb{X})$ of the poset $\mathbb{P}(\mathbb{X})$ is a homogeneous complete Boolean algebra, forcing equivalent to $\mathbb{P}(\mathbb{X})$. All generic extensions by $\mathbb{P}(\mathbb{X})$ are elementarily equivalent.

Proof. By Theorem 2.2 of [6] the poset $\mathbb{P}(\mathbb{X})$ is homogeneous and, by Theorem 2.1. rosq $\mathbb{P}(\mathbb{X})$ is a homogeneousthe algebra. By Fact 1.1 b) the posets $\mathbb{P}(\mathbb{X})$ and $\operatorname{rosq} \mathbb{P}(\mathbb{X})$ are forcing equivalent. By Theorem 4.3 of [6] either $|\operatorname{sq} \mathbb{P}(\mathbb{X})|=1$, and then all generic extensions are trivial, or sq $\mathbb{P}(\mathbb{X})$ is an atomless poset, and then $\mathbb{B}:=\operatorname{rosq} \mathbb{P}(\mathbb{X})$ is an infinite homogeneous complete Boolean algebra. This implies that for each $a, b \in \mathbb{B} \backslash\{0,1\}$ there is $f \in \operatorname{Aut}(\mathbb{B})$ such that $f(a)=b$ (see [4], Proposition 9.13) and, hence $\mathbb{B}^{+}$is a weakly homogeneous partial order (we recall that a partial order $\mathbb{P}=\langle P, \leq\rangle$ is called weakly homogeneous iff for each $p, q \in P$ there is $f \in \operatorname{Aut}(\mathbb{P})$ such that $f(p) \not \perp q)$. By a known fact concerning weakly homogeneous partial orders (see [5], p. 245), for each sentence $\varphi$ of the language of set theory we have $1 \Vdash \varphi$ or $1 \Vdash \neg \varphi$. Thus all generic extensions by $\mathbb{P}(\mathbb{X})$ satisfy the same set of sentences.

\subsection{Forcing-equivalence and isomorphism of Boolean completions}

Here we show that the posets of copies of two structures are forcing equivalent iff their Boolean completions are isomorphic.

Fact 2.6 If $\mathbb{B}$ and $\mathbb{C}$ are complete Boolean algebras such that some $\mathbb{B}$-generic extension is equal to some $\mathbb{C}$-generic extension, then

(a) There are $b \in \mathbb{B}$ and $c \in \mathbb{C}$ such that $b \downarrow \cong c \downarrow$ (see [3], p. 267);

(b) If $\mathbb{B}$ and $\mathbb{C}$ are homogeneous algebras, then $\mathbb{B} \cong \mathbb{C}$. So, $\mathbb{B} \equiv \mathbb{C} \Leftrightarrow \mathbb{B} \cong \mathbb{C}$.

Proof. (b) If $b \in \mathbb{B}$ and $c \in \mathbb{C}$ are the elements from (a), by the homogeneity we have $\mathbb{B} \cong b \downarrow$ and $\mathbb{C} \cong c \downarrow$ and, hence, $\mathbb{B} \cong \mathbb{C}$. 
Theorem 2.7 Let $\mathbb{X}$ and $\mathbb{Y}$ be arbitrary relational structures. Then

(a) $\mathbb{P}(\mathbb{X}) \equiv \mathbb{P}(\mathbb{Y})$ iff rosq $\mathbb{P}(\mathbb{X}) \cong \operatorname{rosq} \mathbb{P}(\mathbb{Y})$;

(b) The collections ge $\mathbb{P}(\mathbb{X})$ and ge $\mathbb{P}(\mathbb{Y})$ are either disjoint or equal.

Proof. (a) By Fact 1.1 b), Fact 2.6 b) and Theorem $2.5, \mathbb{P}(\mathbb{X}) \equiv \mathbb{P}(\mathbb{Y})$ iff ro sq $\mathbb{P}(\mathbb{X})$ $\equiv \operatorname{rosq} \mathbb{P}(\mathbb{Y})$ iff rosq $\mathbb{P}(\mathbb{X}) \cong \operatorname{rosq} \mathbb{P}(\mathbb{Y})$.

(b) If ge $\mathbb{P}(\mathbb{X}) \cap$ ge $\mathbb{P}(\mathbb{Y}) \neq \emptyset$, then by Fact 1.1 b) and Fact 2.6 (b) we have $\operatorname{rosq} \mathbb{P}(\mathbb{X}) \cong \operatorname{rosq} \mathbb{P}(\mathbb{Y})$, which implies ge $(\operatorname{rosq} \mathbb{P}(\mathbb{X}))=\operatorname{ge}(\operatorname{rosq} \mathbb{P}(\mathbb{Y}))$, that is ge $\mathbb{P}(\mathbb{X})=$ ge $\mathbb{P}(\mathbb{Y})$.

\subsection{Isomorphic structures, equimorphic structures}

In this section we prove that the posets of copies of isomorphic (resp. equimorphic) structures are isomorphic (resp. have isomorphic Boolean completions). We will use the following elementary fact.

Fact 2.8 Let $\langle\mathbb{P}, \leq\rangle$ be a pre-order and $p \in \mathbb{P}$. Then

(a) If $G$ is a $\mathbb{P}$-generic filter over $V$ and $p \in G$, then $G \cap p \downarrow$ is a $p \downarrow$-generic filter over $V$ and $V_{\mathbb{P}}[G]=V_{p \downarrow}[G \cap p \downarrow]$;

(b) If $H$ is a $p \downarrow$-generic filter over $V$, then $H \uparrow$ is a $\mathbb{P}$-generic filter over $V$ and $V_{p \downarrow}[H]=V_{\mathbb{P}}[H \uparrow]$.

Lemma 2.9 If $\mathbb{X}$ and $\mathbb{Y}$ are structures of the same language, $h: \mathbb{X} \hookrightarrow \mathbb{Y}$, and $C=h[X]$, then the mapping $F: \mathbb{P}(\mathbb{X}) \rightarrow(C \downarrow)_{\mathbb{P}(\mathbb{X}, \mathbb{Y})}$ defined by $F(A)=h[A]$,

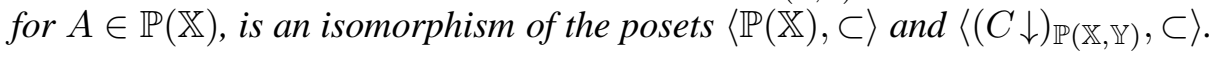

Proof. For $A \in \mathbb{P}(\mathbb{X})$ there is $\varphi: \mathbb{X} \hookrightarrow \mathbb{X}$ such that $\varphi[X]=A$ and, clearly, $h \circ \varphi: \mathbb{X} \hookrightarrow \mathbb{Y}$, thus $h[\varphi[X]]=h[A] \in \mathbb{P}(\mathbb{X}, \mathbb{Y})$ and $h[A] \subset h[X]=C$, which

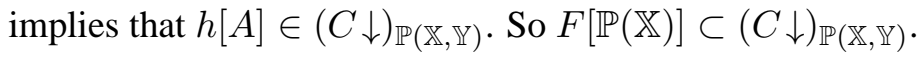

Since $h$ is an injection, for each $A, B \in \mathbb{P}(\mathbb{X})$ we have $F(A) \subset F(B)$ iff $h[A] \subset h[B]$ iff $h^{-1}[h[A]] \subset h^{-1}[h[B]]$ iff $A \subset B$, thus $F$ is an embedding.

If $D \in \mathbb{P}(\mathbb{X}, \mathbb{Y})$ and $D \subset C$, then $h\left[h^{-1}[D]\right]=D$ and the surjective restriction $h \mid h^{-1}[D]: h^{-1}[D] \rightarrow D$ is an isomorphism, which implies $h^{-1}[D] \in \mathbb{P}(\mathbb{X})$. In addition $F\left(h^{-1}[D]\right)=h\left[h^{-1}[D]\right]=D$ thus $F$ is onto.

Theorem 2.10 If $\mathbb{X}$ and $\mathbb{Y}$ are structures of the same relational language, then

(a) $\mathbb{X} \cong \mathbb{Y} \Rightarrow \mathbb{P}(\mathbb{X}) \cong \mathbb{P}(\mathbb{Y})$;

(b) $\mathbb{X} \rightleftarrows \mathbb{Y} \Rightarrow \operatorname{rosq} \mathbb{P}(\mathbb{X}) \cong \operatorname{rosq} \mathbb{P}(\mathbb{Y})$. 
Proof. (a) If $h: \mathbb{X} \rightarrow \mathbb{Y}$ is an isomorphism, then, by Lemma $2.9,\langle\mathbb{P}(\mathbb{X}), \subset\rangle \cong$ $\langle\mathbb{P}(\mathbb{X}, \mathbb{Y}), \subset\rangle$ and, clearly, $\langle\mathbb{P}(\mathbb{X}, \mathbb{Y}), \subset\rangle=\langle\mathbb{P}(\mathbb{Y}), \subset\rangle$.

(b) Let $f: \mathbb{X} \hookrightarrow \mathbb{Y}, g: \mathbb{Y} \hookrightarrow \mathbb{X}$ and $\mathbb{P}(\mathbb{Y}) \uparrow=\{S \subset Y: \exists B \in \mathbb{P}(\mathbb{Y}) B \subset S\}$.

First we show that $\mathbb{P}(\mathbb{X}, \mathbb{Y}):=\{h[X] \mid h: \mathbb{X} \hookrightarrow \mathbb{Y}\}$ is a dense suborder of $\langle\mathbb{P}(\mathbb{Y}) \uparrow, \subset\rangle$. If $C \in \mathbb{P}(\mathbb{X}, \mathbb{Y})$ and $h: \mathbb{X} \hookrightarrow \mathbb{Y}$, where $C=h[X]$, then, clearly, $h \circ g: \mathbb{Y} \hookrightarrow \mathbb{Y}$ and, hence, $h[g[Y]] \in \mathbb{P}(\mathbb{Y})$ and $h[g[Y]] \subset h[X]=C$, which implies $C \in \mathbb{P}(\mathbb{Y}) \uparrow$. Thus $\mathbb{P}(\mathbb{X}, \mathbb{Y}) \subset \mathbb{P}(\mathbb{Y}) \uparrow$. Let $S \in \mathbb{P}(\mathbb{Y}) \uparrow, B \in \mathbb{P}(\mathbb{Y})$, where $B \subset S$ and $\psi: \mathbb{Y} \hookrightarrow \mathbb{Y}$, where $B=\psi[Y]$. Now $\psi \circ f: \mathbb{X} \hookrightarrow \mathbb{Y}$ and, hence, $\psi[f[X]] \in \mathbb{P}(\mathbb{X}, \mathbb{Y})$ and $\psi[f[X]] \subset \psi[Y]=B \subset S$. Thus $\mathbb{P}(\mathbb{X}, \mathbb{Y})$ is dense in $\langle\mathbb{P}(\mathbb{Y}) \uparrow, \subset\rangle$. Since $\mathbb{P}(\mathbb{Y})$ is dense in $\langle\mathbb{P}(\mathbb{Y}) \uparrow, \subset\rangle$ as well, we have

$$
\langle\mathbb{P}(\mathbb{X}, \mathbb{Y}), \subset\rangle \equiv\langle\mathbb{P}(\mathbb{Y}), \subset\rangle .
$$

Now let $W$ be a generic extension by $\mathbb{P}(\mathbb{Y})$. By (3) $W=V_{\mathbb{P}(\mathbb{X}, \mathbb{Y})}[G]$, where $G$ is a $\mathbb{P}(\mathbb{X}, \mathbb{Y})$-generic filter over $V$. Let $C \in G$. By Fact 2.8(a) we have $V_{\mathbb{P}(\mathbb{X}, \mathbb{Y})}[G]=$

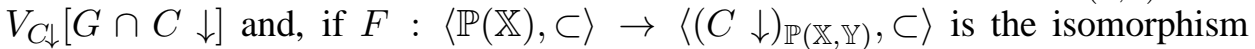
defined in Lemma 2.9, then $H:=F^{-1}[G \cap C \downarrow]$ is a $\mathbb{P}(\mathbb{X})$-generic filter over $V$ and $V_{C \downarrow}[G \cap C \downarrow]=V_{\mathbb{P}(\mathbb{X})}[H]$. Thus $W=V_{\mathbb{P}(\mathbb{X})}[H]$ and, by Theorem 2.7 b), $\mathbb{P}(\mathbb{X}) \equiv \mathbb{P}(\mathbb{Y})$. Now, by Theorem 2.7 a), rosq $\mathbb{P}(\mathbb{X}) \cong \operatorname{rosq} \mathbb{P}(\mathbb{Y})$.

\section{The hierarchy of similarities on the class $\operatorname{Mod}_{L}(\mathrm{X})$}

Now we restrict our consideration to some smaller classes of structures. If $L=$ $\left\langle R_{i}: i \in I\right\rangle$ is a language, $X$ a fixed set and $\rho=\left\langle\rho_{i}: i \in I\right\rangle \in \operatorname{Int}_{L}(X)$, we will abuse notation writing $\mathbb{P}(\rho)$ instead of $\mathbb{P}(\langle X, \rho\rangle)$ and $\langle\mathbb{P}(\langle X, \rho\rangle), \subset\rangle$ whenever the context admits it. So, restricting our similarity relations to the set $\operatorname{Mod}_{L}(X)$ or, equivalently, to the corresponding set of interpretations, $\operatorname{Int}_{L}(X)$, we obtain the following equivalence relations: for $\rho=\left\langle\rho_{i}: i \in I\right\rangle, \sigma=\left\langle\sigma_{i}: i \in I\right\rangle \in \operatorname{Int}_{L}(X)$ (writing $\rho \cong \sigma$ instead of $\langle X, \rho\rangle \cong\langle X, \sigma\rangle$ and similarly for $\rho \rightleftarrows \sigma$ ) we define

$$
\begin{array}{lll}
\rho \sim_{0} \sigma \Leftrightarrow \rho=\sigma & & \rho \sim_{6} \sigma \Leftrightarrow \mathbb{P}(\rho) \cong \mathbb{P}(\sigma) \\
\rho \sim_{1} \sigma \Leftrightarrow \mathbb{P}(\rho)=\mathbb{P}(\sigma) \wedge \rho \cong \sigma & & \rho \sim_{7} \sigma \Leftrightarrow \operatorname{sq} \mathbb{P}(\rho) \cong \operatorname{sq} \mathbb{P}(\sigma) \wedge \rho \rightleftarrows \sigma \\
\rho \sim_{2} \sigma \Leftrightarrow \mathbb{P}(\rho)=\mathbb{P}(\sigma) \wedge \rho \rightleftarrows \sigma & & \rho \sim_{8} \sigma \Leftrightarrow \operatorname{sq} \mathbb{P}(\rho) \cong \operatorname{sq} \mathbb{P}(\sigma) \\
\rho \sim_{3} \sigma \Leftrightarrow \rho \cong \sigma & & \rho \sim_{9} \sigma \Leftrightarrow \rho \rightleftarrows \sigma \\
\rho \sim_{4} \sigma \Leftrightarrow \mathbb{P}(\rho)=\mathbb{P}(\sigma) & & \rho \sim_{10} \sigma \Leftrightarrow \mathbb{P}(\rho) \equiv \mathbb{P}(\sigma) \\
\rho \sim_{5} \sigma \Leftrightarrow \mathbb{P}(\rho) \cong \mathbb{P}(\sigma) \wedge \rho \rightleftarrows \sigma & & \rho \sim_{11} \sigma \Leftrightarrow 0=0 .
\end{array}
$$

Then some implications between the similarities on the set $\operatorname{Mod}_{L}(X)$ are displayed in Figure 2

It is natural to ask are there more implications in it (except the ones which follow from the transitivity), that is, are some of the implications $a-o$, in fact, 


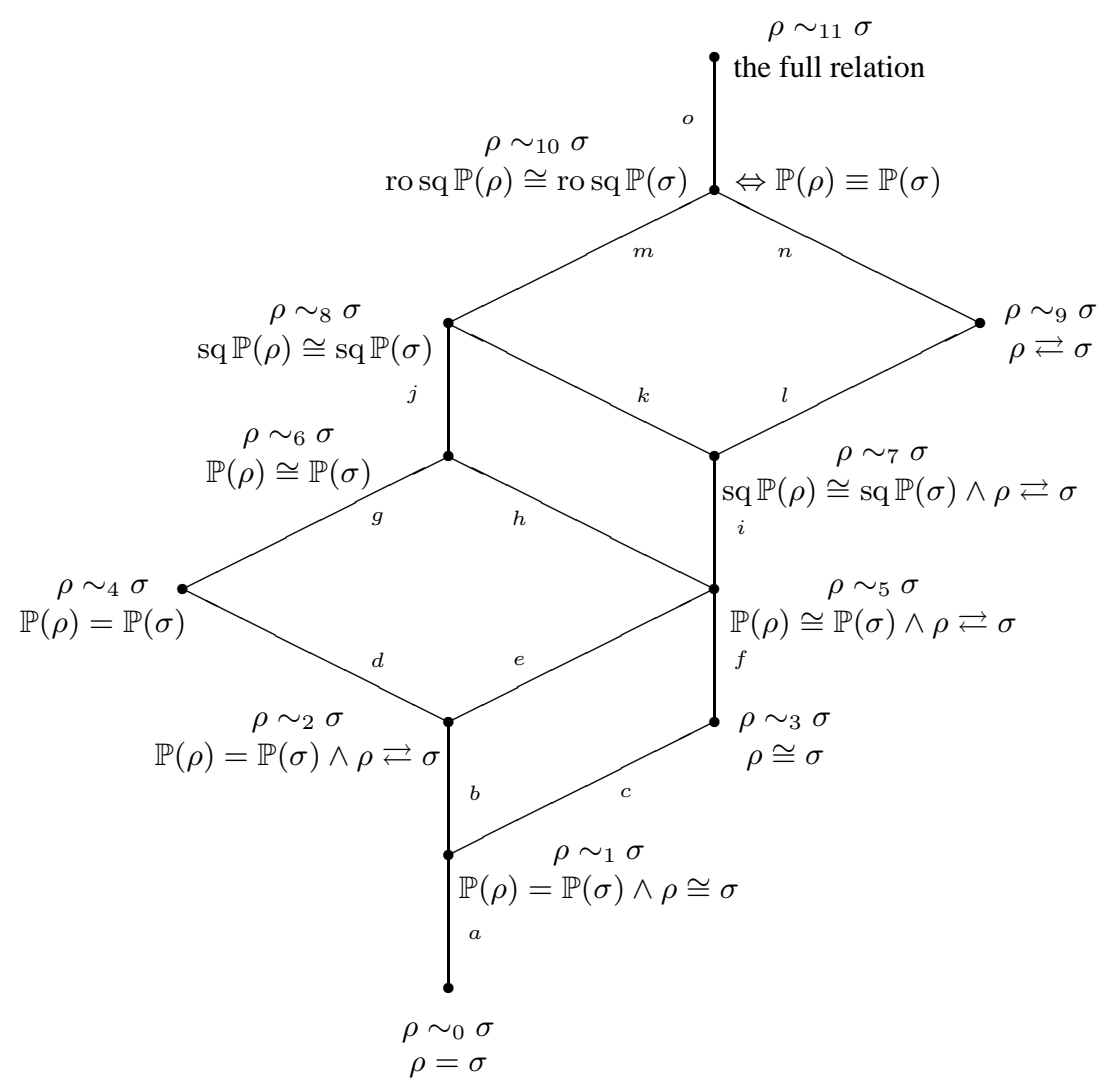

Figure 2: Some implications between the similarities on $\operatorname{Mod}_{L}(X)$

equivalences. Concerning this question we will show that the class of all relational structures splits into the following three parts: finite structures, infinite structures of unary languages, and infinite structures of non-unary languages. (A language $L=\left\langle R_{i}: i \in I\right\rangle$ is called unary iff $\operatorname{ar}\left(R_{i}\right)=1$, for all $i \in I$. Structures of unary languages will be called unary structures). Let us call a class $\mathcal{C}$ of structures a Cantor-Schröder-Bernstein (CSB) class iff

$$
\forall \mathbb{X}, \mathbb{Y} \in \mathcal{C}(\mathbb{X} \rightleftarrows \mathbb{Y} \Rightarrow \mathbb{X} \cong \mathbb{Y})
$$

For finite structures the diagram from Figure 2 collapses significantly.

Example 3.1 If $L$ is an arbitrary relational language and $X$ a finite set, then for each $\rho \in \operatorname{Int}_{L}(X)$ we have $\mathbb{P}(\rho)=\{X\}$, because $X \in \mathbb{P}(X, \rho) \subset[X]^{|X|}=$ $\{X\}$. Thus, $\sim_{4}$ is the full relation, which implies that $\sim_{4}=\sim_{6}=\sim_{8}=\sim_{10}=\sim_{11}$. 
In addition, $\operatorname{Mod}_{L}(X)$ is a CSB class. Namely, if $\rho, \sigma \in \operatorname{Int}_{L}(X)$ and $\rho \rightleftarrows$ $\sigma$, then there is an embedding $f:\langle X, \rho\rangle \rightarrow\langle X, \sigma\rangle$, and, since $X$ is a finite set, $f$ is an isomorphism, thus $\rho \cong \sigma$. So we have $\sim_{9} \subset \sim_{1}$, which implies $\sim_{1}=\sim_{2}=\sim_{3}=\sim_{5}=\sim_{7}=\sim_{9}$. Since $\langle X,\langle\emptyset, \emptyset, \ldots\rangle\rangle \neq\left\langle X,\left\langle X^{n_{i}}: i \in I\right\rangle\right\rangle$, we have $\sim_{3} \neq \sim_{11}$. If $|X|>1$, let $a$ and $b$ be different elements of $X, i_{0} \in I$, and let $\rho, \sigma \in \operatorname{Int}_{L}(X)$, where $\rho_{i_{0}}=\{\langle a, a, \ldots, a\rangle\}, \sigma_{i_{0}}=\{\langle b, b, \ldots, b\rangle\} \subset X^{n_{i_{0}}}$ and $\rho_{i}=\sigma_{i}=\emptyset$, for $i \neq i_{0}$. Then $\rho \neq \neq_{0} \sigma$, but $\rho \cong \sigma$ and, hence, $\sim_{0} \neq \sim_{1}$. Thus Figure 3 describes the hierarchy of the similarities $\sim_{k}$ on the set $\operatorname{Mod}_{L}(X)$, if $|X|>1$.

We prove that $\sim_{0}=\sim_{1} \Leftrightarrow|X|=1$. Let $X=\{x\}$ and $\rho, \sigma \in \operatorname{Int}_{L}(X)$, where $\rho \sim_{1} \sigma$. Then there is an isomorphism $f:\langle\{x\}, \rho\rangle \rightarrow\langle\{x\}, \sigma\rangle$ and, consequently, for each $i \in I$ we have $\langle x, x, \ldots, x\rangle \in \rho_{i} \Leftrightarrow\langle x, x, \ldots, x\rangle \in \sigma_{i}$ and, hence, $\rho_{i}=\sigma_{i}$. So $\rho=\sigma$, that is $\rho \sim_{0} \sigma$ and the inclusion $\sim_{1} \subset \sim_{0}$ is proved.

$$
\left\{\begin{array}{l}
\sim_{4}=\sim_{6}=\sim_{8}=\sim_{10}=\sim_{11}=\text { the full relation } \\
\sim_{1}=\sim_{2}=\sim_{3}=\sim_{5}=\sim_{7}=\sim_{9}=\text { the isomorphism } \\
\sim_{0}=\text { the equality }
\end{array}\right.
$$

Figure 3: The similarities on the class $\operatorname{Mod}_{L}(X)$, if $1<|X|<\omega$

\subsection{Infinite unary structures}

In this subsection we assume that $L=\left\langle R_{i}: i \in I\right\rangle$ is a unary relational language. If $\mathbb{X}=\left\langle X,\left\langle\rho_{i}: i \in I\right\rangle\right\rangle$ is an $L$-structure, it is easy to check that the binary relation $\approx$ on the set $X$ defined by: $x \approx y \Leftrightarrow \forall i \in I \quad\left(x \in \rho_{i} \Leftrightarrow y \in \rho_{i}\right)$ is an equivalence relation. Then $[x]:=\{y \in X: y \approx x\}$ is the equivalence class of $x \in X$, and if $X / \approx=\left\{X_{j}: j \in J\right\}$ is the corresponding partition we define $\kappa_{j}:=\left|X_{j}\right|$, for $j \in J$, and $J_{0}:=\left\{j \in J:\left|X_{j}\right|<\omega\right\}$.

Theorem 3.2 Let $\mathbb{X}=\left\langle X,\left\langle\rho_{i}: i \in I\right\rangle\right\rangle$ be a unary structure. Then

(a) If $f: X \rightarrow X$ is an injection, then $f \in \operatorname{Emb}(\mathbb{X}) \Leftrightarrow \forall x \in X \quad f[[x]] \subset[x]$;

(b) If $J_{0}=J$, then $\mathbb{P}(\mathbb{X})=\{X\}$;

(c) If $J_{0} \neq J$, then the poset $\mathbb{P}(\mathbb{X})$ is atomless and we have

$$
\mathbb{P}(\mathbb{X}) \cong \prod_{j \in J \backslash J_{0}}\left\langle\left[\kappa_{j}\right]^{\kappa_{j}}, \subset\right\rangle \text { and } \mathrm{sq} \mathbb{P}(\mathbb{X}) \cong \prod_{j \in J \backslash J_{0}}\left(P\left(\kappa_{j}\right) /\left[\kappa_{j}\right]^{<\kappa_{j}}\right)^{+} \text {. }
$$


Proof. (a) If $f \in \operatorname{Emb}(\mathbb{X})$ and $x \in X$, then $x \in \rho_{i} \Leftrightarrow f(x) \in \rho_{i}$, for each $i \in I$, thus $x \approx f(x)$. So for $y \in[x]$ we have $f(y) \approx y \approx x$ and, hence, $f(y) \in[x]$.

Let $f[[x]] \subset[x]$, for all $x \in X$. Then, for $x \in X, x \in[x]$ implies $f(x) \in[x]$, that is $f(x) \approx x$. Hence $\forall i \in I \forall x \in X\left(x \in \rho_{i} \Leftrightarrow f(x) \in \rho_{i}\right)$, so $f \in \operatorname{Emb}(\mathbb{X})$.

(b) Let $J_{0}=J$. By (a), for $f \in \operatorname{Emb}(\mathbb{X})$ and $x \in X$ we have $f[[x]] \subset[x]$ and, since $|[x]|<\omega, f[[x]]=[x]$, which implies $f[X]=X$.

(c) If $f \in \operatorname{Emb}(\mathbb{X})$, then, by (a), $f\left[X_{j}\right]=X_{j}$, for all $j \in J_{0}$, and $C_{j}:=$ $f\left[X_{j}\right] \in\left[X_{j}\right]^{\kappa_{j}}$, for all $j \in J \backslash J_{0}$. Thus the inclusion " $\subset$ " in the equality

$$
\mathbb{P}(\mathbb{X})=\left\{\bigcup_{j \in J_{0}} X_{j} \cup \bigcup_{j \in J \backslash J_{0}} C_{j}:\left\langle C_{j}: j \in J \backslash J_{0}\right\rangle \in \prod_{j \in J \backslash J_{0}}\left[X_{j}\right]^{\kappa_{j}}\right\}
$$

is proved. On the other hand, if $\left\langle C_{j}: j \in J \backslash J_{0}\right\rangle \in \prod_{j \in J \backslash J_{0}}\left[X_{j}\right]^{\kappa_{j}}$ and if we choose bijections $\varphi_{j}: X_{j} \rightarrow C_{j}$, for all $j \in J \backslash J_{0}$, then by (a) we have $f=\bigcup_{j \in J_{0}} \operatorname{id}_{X_{j}} \cup \bigcup_{j \in J \backslash J_{0}} \varphi_{j} \in \operatorname{Emb}(\mathbb{X})$ and, hence, $\bigcup_{j \in J_{0}} X_{j} \cup \bigcup_{j \in J \backslash J_{0}} C_{j} \in$ $\mathbb{P}(\mathbb{X})$, so $(4)$ is true. Thus the mapping $F: \prod_{j \in J \backslash J_{0}}\left\langle\left[X_{j}\right]^{\kappa_{j}}, \subset\right\rangle \rightarrow\langle\mathbb{P}(\mathbb{X}), \subset\rangle$ given by

$$
F\left(\left\langle C_{j}: j \in J \backslash J_{0}\right\rangle\right)=\bigcup_{j \in J_{0}} X_{j} \cup \bigcup_{j \in J \backslash J_{0}} C_{j}
$$

is a well-defined surjection and, since $\left\{X_{j}: j \in J\right\}$ is a partition of $X$, it is an injection. It is easy to see that $F$ is an order isomorphism. By Fact 1.1 (c) we have $\operatorname{sq}\langle\mathbb{P}(\mathbb{X}), \subset\rangle \cong \prod_{j \in J \backslash J_{0}} \operatorname{sq}\left\langle\left[\kappa_{j}\right]^{\kappa_{j}}, \subset\right\rangle=\prod_{j \in J \backslash J_{0}}\left(P\left(\kappa_{j}\right) /\left[\kappa_{j}\right]^{<\kappa_{j}}\right)^{+}$.

Lemma 3.3 Let $\kappa \geq \omega$ be a cardinal, $U \subset \kappa$ and $\lambda:=\min \{|U|,|\kappa \backslash U|\}$. Then $\rho=\langle U, \emptyset, \emptyset, \ldots\rangle \in \operatorname{Int}_{L}(\kappa)$ and we have

(a) $\mathbb{P}(\rho)=\left\{C_{1} \cup C_{2}: C_{1} \in[U]^{|U|} \wedge C_{2} \in[\kappa \backslash U]^{|\kappa \backslash U|}\right\}$;

(b) $\mathbb{P}(\rho) \cong\left\langle[\kappa]^{\kappa}, \subset\right\rangle \times\left\langle[\lambda]^{\lambda}, \subset\right\rangle$;

(c) $\operatorname{sq} \mathbb{P}(\rho) \cong\left(P(\kappa) /[\kappa]^{<\kappa}\right)^{+} \times\left(P(\lambda) /[\lambda]^{<\lambda}\right)^{+}$, where, by convention, for $\lambda \in \omega$, by $\left(P(\lambda) /[\lambda]^{<\lambda}\right)^{+}$we denote the one-element poset.

Proof. For $x, y \in \kappa$ we have: $x \approx y$ iff $x \in \rho_{i} \Leftrightarrow y \in \rho_{i}$, for all $i \in I$, iff $x \in U \Leftrightarrow y \in U$. Thus $\kappa / \approx=\{U, \kappa \backslash U\}$ and we apply Theorem 3.2

Fact 3.4 (a) If $\kappa>\omega$ is a regular cardinal and $2^{\kappa}=\kappa^{+}$, then $\operatorname{ro}\left(P(\kappa) /[\kappa]^{<\kappa}\right) \cong$ $\operatorname{Col}\left(\omega, 2^{\kappa}\right)$ (Balcar, Vopěnka [1]; see also [2], p. 380).

(b) Under $\mathrm{CH}$, all separative atomless $\omega_{1}$-closed posets of size $\omega_{1}$ are forcing equivalent (for example to $\mathrm{Col}\left(\omega_{1}, \omega_{1}\right)$ ) (folklore).

(c) If $\lambda>\omega$ is a cardinal and $\mathbb{P}$ a poset of size $\lambda$ such that $1_{\mathbb{P}} \Vdash|\check{\lambda}|=\check{\omega}$, then ro $\mathbb{P} \cong \operatorname{Col}(\omega, \lambda)$ (see [3], p. 277).

(d) If $\mathbb{B}$ is a Boolean algebra of size $>2$, then $\mathbb{B}^{+} \neq \mathbb{B}^{+} \times \mathbb{B}^{+}$. 
Proof. (d) The sentence $\forall x \neq 1 \exists_{1} y(x \perp y \& x \vee y=1)$ is true in the poset $\mathbb{B}^{+}$, but it is not true in $\mathbb{B}^{+} \times \mathbb{B}^{+}$. Namely, since $|\mathbb{B}|>2$, there is $a \in \mathbb{B}^{+} \backslash\{1\}$ and we have $x:=\langle 1, a\rangle \in\left(\mathbb{B}^{+} \times \mathbb{B}^{+}\right) \backslash\{\langle 1,1\rangle\}$ and $a^{\prime} \in \mathbb{B}^{+}$, but for each $b \in \mathbb{B}^{+}$we have $\langle 1, a\rangle \perp\left\langle b, a^{\prime}\right\rangle$ and $\langle 1, a\rangle \vee\left\langle b, a^{\prime}\right\rangle=\langle 1,1\rangle$.

Theorem 3.5 For any unary language $L$ and infinite cardinal $\kappa$ we have

(a) $\operatorname{Mod}_{L}(\kappa)$ is a CSB class;

(b) Figure 4 describes the hierarchy of the similarities $\sim_{k}$, for $k \neq 8,10$, on the set $\operatorname{Mod}_{L}(\kappa)$. In addition we have $\sim_{8} \neq \sim_{11}$.

(c) If $\kappa$ is a regular cardinal and $2^{\kappa}=\kappa^{+}$, then $\sim_{8} \neq \sim_{10}$.

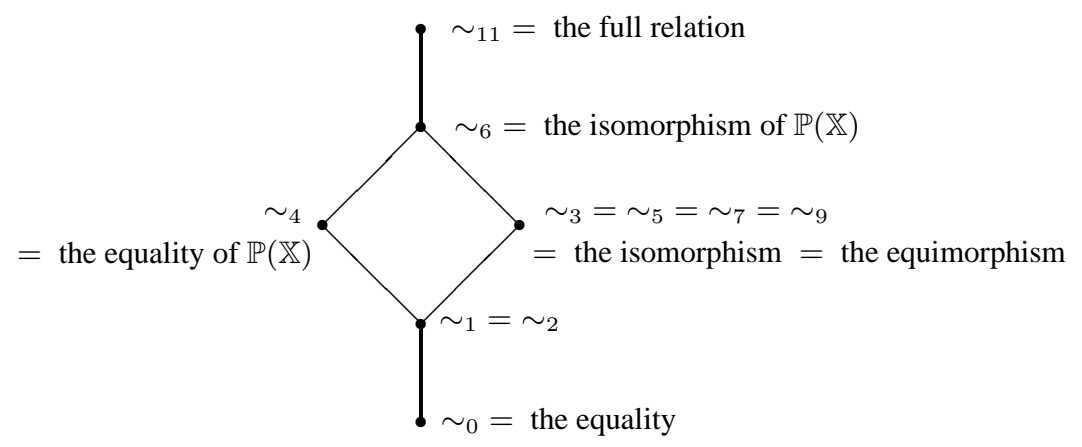

Figure 4: The similarities on $\operatorname{Mod}_{L}(\kappa)$, for unary $L$ and infinite $\kappa$

Proof. Let $L=\left\{R_{i}: i \in I\right\}$.

(a) Assuming that $\rho=\left\langle\rho_{i}: i \in I\right\rangle, \sigma=\left\langle\sigma_{i}: i \in I\right\rangle \in \operatorname{Int}_{L}(\kappa)$ and $\rho \rightleftarrows \sigma$ we show that $\rho \cong \sigma$. By the assumption, there are embeddings

$$
f:\langle\kappa, \rho\rangle \hookrightarrow\langle\kappa, \sigma\rangle \text { and } g:\langle\kappa, \sigma\rangle \hookrightarrow\langle\kappa, \rho\rangle .
$$

Let $\approx_{\rho}$ and $\approx_{\sigma}$ be the equivalence relations determined by the interpretations $\rho$ and $\sigma$ respectively (see Theorem 3.2 ) and, for $x \in \kappa$, let $[x]_{\rho}$ and $[x]_{\sigma}$ be the corresponding equivalence classes. First we prove that

$$
\forall x \in \kappa \quad f\left[[x]_{\rho}\right] \subset[f(x)]_{\sigma} \text { and } \forall x \in \kappa \quad g\left[[x]_{\sigma}\right] \subset[g(x)]_{\rho} .
$$

For a proof of the first statement we take $x \in \kappa$ and $y \in[x]_{\rho}$. Then $y \approx_{\rho} x$, that is

$$
\forall i \in I \quad\left(x \in \rho_{i} \Leftrightarrow y \in \rho_{i}\right),
$$


and, since $f$ is an embedding, we have

$$
\forall i \in I \quad \forall x \in \kappa \quad\left(x \in \rho_{i} \Leftrightarrow f(x) \in \sigma_{i}\right) .
$$

We prove that $f(y) \in[f(x)]_{\sigma}$, which means that $f(y) \in \sigma_{i} \Leftrightarrow f(x) \in \sigma_{i}$, for all $i \in I$. So, $f(y) \in \sigma_{i}$ iff (by (8)) $y \in \rho_{i}$ iff (by (7)) $x \in \rho_{i}$ iff (by (8) $f(x) \in \sigma_{i}$. Thus the first statement of (6) is proved and the second has a symmetric proof.

Let $\kappa=\bigcup_{j \in J} X_{j}$ and $\kappa=\bigcup_{k \in K} Y_{k}$ be the partitions determined by the relations $\approx_{\rho}$ and $\approx_{\sigma}$ respectively. By (6), if $j \in J$ and $X_{j}=[x]_{\rho}$, then $f\left[X_{j}\right] \subset$ $[f(x)]_{\sigma}=Y_{k}$, for (a unique) $k \in K$. Similarly, for each $k \in K$ there is a unique $j \in J$ satisfying $g\left[Y_{k}\right] \subset X_{j}$ so we define the functions

$$
\begin{aligned}
& F: J \rightarrow K \text { by: } \quad F(j)=k \text { iff } f\left[X_{j}\right] \subset Y_{k}, \\
& G: K \rightarrow J \text { by: } G(k)=j \text { iff } g\left[Y_{k}\right] \subset X_{j},
\end{aligned}
$$

and prove that

$$
G \circ F=\mathrm{id}_{J} \quad \text { and } F \circ G=\mathrm{id}_{K} .
$$

By (5) we have $g \circ f:\langle\kappa, \rho\rangle \hookrightarrow\langle\kappa, \rho\rangle$ and, by Theorem 3.2(a),

$$
\forall x \in X \quad g\left[f\left[[x]_{\rho}\right]\right] \subset[x]_{\rho} .
$$

For $j \in J$ we prove that $G(F(j))=j$. Let $F(j)=k$ and $x \in X_{j}$. Then $X_{j}=[x]_{\rho}$, by (6) $f\left[X_{j}\right]=f\left[[x]_{\rho}\right] \subset[f(x)]_{\sigma}=Y_{k^{\prime}}$, for some $k^{\prime} \in K$, and, by (9) $f\left[X_{j}\right] \subset Y_{k}$, which implies $k^{\prime}=k$. Thus $f\left[[x]_{\rho}\right] \subset Y_{k}$ and, hence,

$$
g\left[f\left[[x]_{\rho}\right]\right] \subset g\left[Y_{k}\right]
$$

Let $G(k)=j^{\prime}$. Then by (10) and (13) we have $g\left[f\left[[x]_{\rho}\right]\right] \subset g\left[Y_{k}\right] \subset X_{j^{\prime}}$ and, by (12), $g\left[f\left[[x]_{\rho}\right]\right] \subset X_{j}$, which implies $j^{\prime}=j$. Thus $G(F(j))=G(k)=j$ and the first equality in (11) is proved. The second equality has a similar proof.

Now we prove that

$$
\forall j \in J \quad\left|X_{j}\right|=\left|Y_{F(j)}\right| \cdot
$$

By (9) we have $\left|X_{j}\right|=\left|f\left[X_{j}\right]\right| \leq\left|Y_{F(j)}\right|$ and, by (10) and (11), $\left|Y_{F(j)}\right|=$ $\left|g\left[Y_{F(j)}\right]\right| \leq\left|X_{G(F(j))}\right|=\left|X_{j}\right|$. So (14) is true.

By (14) there are bijections $\varphi_{j}: X_{j} \rightarrow Y_{F(j)}$; let $\varphi=\bigcup_{j \in J} \varphi_{j}: \kappa \rightarrow \kappa$. Since $\left\{X_{j}: j \in J\right\}$ is a partition of $\kappa$ the mapping $\varphi$ is well defined. By (11) $F: J \rightarrow K$ is a bijection and, since the mappings $\varphi_{j}$ are surjections, $\varphi$ is a surjection as well. Since $\left\{Y_{k}: k \in K\right\}$ is a partition of $\kappa$ and the mappings $\varphi_{j}$ are injections, $\varphi$ is a injection too. Thus $\varphi$ is a bijection from $\kappa$ onto $\kappa$. 
In order to show that $\varphi:\langle\kappa, \rho\rangle \rightarrow\langle\kappa, \sigma\rangle$ is an isomorphism, that is

$$
\forall i \in I \quad \forall x \in \kappa \quad\left(x \in \rho_{i} \Leftrightarrow \varphi(x) \in \sigma_{i}\right),
$$

we take $i_{0} \in I$ and $x_{0} \in \kappa$. Let $j \in J$, where $x_{0} \in X_{j}$. Then $X_{j}=\left[x_{0}\right]_{\rho}$ and $\varphi\left(x_{0}\right)=\varphi_{j}\left(x_{0}\right) \in Y_{F(j)}$ and, by (6) and (9), $f\left(x_{0}\right) \in\left[f\left(x_{0}\right)\right]_{\sigma}=Y_{F(j)}$. Thus $\varphi\left(x_{0}\right) \approx_{\sigma} f\left(x_{0}\right)$, that is

$$
\forall i \in I \quad\left(\varphi\left(x_{0}\right) \in \sigma_{i} \Leftrightarrow f\left(x_{0}\right) \in \sigma_{i}\right) .
$$

Now $x_{0} \in \rho_{i_{0}}$ iff (by (8)) $f\left(x_{0}\right) \in \sigma_{i_{0}}$ iff (by (16) $) \varphi\left(x_{0}\right) \in \sigma_{i_{0}}$ and (15) is proved. Thus $\varphi:\langle\kappa, \rho\rangle \rightarrow\langle\kappa, \sigma\rangle$ is an isomorphism and, hence, $\rho \cong \sigma$.

(b) By (a) we have $\sim_{9} \subset \sim_{3}$ which, according to Figure 2, implies that $\sim_{3}=$ $\sim_{5}=\sim_{7}=\sim_{9}$ and $\sim_{1}=\sim_{2}$.

Let us prove that $\sim_{0} \varsubsetneqq \sim_{1}$. If $\kappa=A \cup B$, where $A \cap B=\emptyset$ and $A, B \in[\kappa]^{\kappa}$, then $\rho:=\langle A, \emptyset, \emptyset, \ldots\rangle \neq \sigma:=\langle B, \emptyset, \emptyset, \ldots\rangle$. By Lemma3.3 a) we have $\mathbb{P}(\rho)=$ $\left\{C_{1} \cup C_{2}: C_{1} \in[A]^{\kappa} \wedge C_{2} \in[B]^{\kappa}\right\}=\mathbb{P}(\sigma)$. If $f: \kappa \rightarrow \kappa$ is a bijection satisfying $f[A]=B$, then $f:\langle\kappa, \rho\rangle \rightarrow\langle\kappa, \sigma\rangle$ is an isomorphism and, hence, $\rho \sim_{1} \sigma$, but $\rho \chi_{0} \sigma$.

Now we prove that $\sim_{3} \not \subset \sim_{4}$ and, hence, $\sim_{1} \varsubsetneqq \sim_{3}$ and $\sim_{4} \varsubsetneqq \sim_{6}$. Let $x, y \in \kappa$, $x \neq y$ and let $\rho:=\langle\{x\}, \emptyset, \emptyset, \ldots\rangle$ and $\sigma:=\langle\{y\}, \emptyset, \emptyset, \ldots\rangle$. If $f: \kappa \rightarrow \kappa$ is a bijection satisfying $f(x)=y$, then $f:\langle\kappa, \rho\rangle \rightarrow\langle\kappa, \sigma\rangle$ is an isomorphism and, hence, $\rho \sim_{3} \sigma$. By Lemma 3.3 (a) we have

$$
\mathbb{P}(\rho)=\left\{C_{1} \cup C_{2}: C_{1} \in[\{x\}]^{1} \wedge C_{2} \in[\kappa \backslash\{x\}]^{\kappa}\right\}=\left\{C \in[\kappa]^{\kappa}: x \in C\right\}
$$

and, similarly, $\mathbb{P}(\sigma)=\left\{C \in[\kappa]^{\kappa}: y \in C\right\}$, which implies that $\kappa \backslash\{y\} \in$ $\mathbb{P}(\rho) \backslash \mathbb{P}(\sigma)$. Thus $\rho \chi_{4} \sigma$.

Further we prove that $\sim_{4} \not \subset \sim_{3}$ and, hence, $\sim_{1} \varsubsetneqq \sim_{4}$ and $\sim_{3} \varsubsetneqq \sim_{6}$. Let $x \in \kappa$ and $\rho:=\langle\{x\}, \emptyset, \emptyset, \ldots\rangle$ and $\sigma:=\langle\kappa \backslash\{x\}, \emptyset, \emptyset, \ldots\rangle$. Then, clearly, $\rho \neq \sigma$, that is $\rho \chi_{3} \sigma$. As above we have $\mathbb{P}(\rho)=\left\{C \in[\kappa]^{\kappa}: x \in C\right\}$ and, by Lemma 3.3 (a), $\mathbb{P}(\sigma)=\left\{C_{1} \cup C_{2}: C_{1} \in[\kappa \backslash\{x\}]^{\kappa} \wedge C_{2} \in[\{x\}]^{1}\right\}=\left\{C \in[\kappa]^{\kappa}: x \in C\right\}=\mathbb{P}(\rho)$. Thus $\rho \sim_{4} \sigma$.

Finally we prove that $\sim_{8} \neq \sim_{11}$, which implies $\sim_{6} \neq \sim_{11}$. Let $U \subset \kappa$, where $|U|=|\kappa \backslash U|=\kappa$ and let $\rho:=\langle\emptyset, \emptyset, \emptyset, \ldots\rangle$ and $\sigma:=\langle U, \emptyset, \emptyset, \ldots\rangle$. Then, by Lemma 3.3 (b) and (c), $\mathbb{P}(\rho)=\left\langle[\kappa]^{\kappa}, \subset\right\rangle, \mathbb{P}(\sigma)=\left\langle[\kappa]^{\kappa}, \subset\right\rangle \times\left\langle[\kappa]^{\kappa}, \subset\right\rangle$, and

$$
\begin{gathered}
\operatorname{sq} \mathbb{P}(\rho) \cong\left(P(\kappa) /[\kappa]^{<\kappa}\right)^{+}, \\
\operatorname{sq} \mathbb{P}(\sigma) \cong\left(P(\kappa) /[\kappa]^{<\kappa}\right)^{+} \times\left(P(\kappa) /[\kappa]^{<\kappa}\right)^{+} .
\end{gathered}
$$

By Fact $3.4(\mathrm{~d})$, the poset $\left(P(\kappa) /[\kappa]^{<\kappa}\right)^{+}$is not isomorphic to its square. So, by (17) and (18) we have $\rho \nsim_{8} \sigma$. 
(c) For $\rho$ and $\sigma$ defined in the previous paragraph we have $\rho \chi_{8} \sigma$ and we prove that $\rho \sim_{10} \sigma$. First we consider the case when $\kappa>\omega$. By (17) and Fact 3.4, $\mathbb{P}(\rho) \equiv\left(\operatorname{Col}\left(\omega, 2^{\kappa}\right)\right)^{+}$. By $[18)$, forcing by the poset sq $\mathbb{P}(\sigma)$ collapses $2^{\kappa}$ to $\omega$ and, since the poset is of size $2^{\kappa}$, by Fact 3.4 (c) we have rosq $\mathbb{P}(\sigma) \cong \operatorname{Col}\left(\omega, 2^{\kappa}\right)$. Thus the posets $\mathbb{P}(\rho)$ and $\mathbb{P}(\sigma)$ are forcing equivalent, that is $\rho \sim_{10} \sigma$. If $\kappa=\omega$ we use Fact 3.4(b).

The following theorem shows that the equivalence of the similarities $\sim_{8}$ (the isomorphism of sq $\mathbb{P}(\mathbb{X})$ ) and $\sim_{10}$ (the isomorphism of rosq $\mathbb{P}(\mathbb{X})$ ) is independent of $\mathrm{ZFC}$ even for the simplest unary language.

Theorem 3.6 If $L$ is the language containing only one unary relational symbol, then on $\operatorname{Mod}_{L}(\omega)$ we have $\sim_{8}=\sim_{6}$ and

$$
\sim_{10}= \begin{cases}\sim_{11} & \text { if the poset }(P(\omega) / \text { Fin })^{+} \text {is forcing equivalent to its square } \\ \sim_{6} & \text { otherwise. }\end{cases}
$$

So, the equality $\sim_{8}=\sim_{10}$ is independent of $Z F C$.

Proof. By Lemma 3.3, for $U \subset \omega$, writing $\mathbb{P}(U)$ instead of $\langle\mathbb{P}(\omega, U), \subset\rangle$, we have

$$
\begin{gathered}
\mathbb{P}(U) \cong \begin{cases}\left\langle[\omega]^{\omega}, \subset\right\rangle & \text { if }|U|<\omega \text { or }|\omega \backslash U|<\omega, \\
\left\langle[\omega]^{\omega}, \subset\right\rangle^{2} & \text { otherwise; }\end{cases} \\
\text { sq } \mathbb{P}(U) \cong \begin{cases}(P(\omega) / \text { Fin })^{+} & \text {if }|U|<\omega \text { or }|\omega \backslash U|<\omega, \\
\left((P(\omega) / \text { Fin })^{+}\right)^{2} & \text { otherwise. }\end{cases}
\end{gathered}
$$

If $U_{1}, U_{2} \subset \omega$ and $U_{1} \not{ }_{6} U_{2}$, that is $\mathbb{P}\left(U_{1}\right) \neq \mathbb{P}\left(U_{2}\right)$, then, by (19) and (20), for example, sq $\mathbb{P}\left(U_{1}\right) \cong(P(\omega) / \text { Fin })^{+}$and sq $\mathbb{P}\left(U_{2}\right) \cong\left((P(\omega) / \text { Fin })^{+}\right)^{2}$ and, by Fact $3.4(\mathrm{~d})$, sq $\mathbb{P}\left(U_{1}\right) \nRightarrow \mathrm{sq} \mathbb{P}\left(U_{2}\right)$, that is $U_{1} \chi_{8} U_{2}$. Thus $\sim_{8} \subset \sim_{6}$, which implies $\sim_{8}=\sim_{6}$.

If $(P(\omega) / \text { Fin })^{+} \equiv\left((P(\omega) / \text { Fin })^{+}\right)^{2}$, then by (20) for each $U \subset \omega$ we have $\mathbb{P}(U) \equiv(P(\omega) / \text { Fin })^{+}$and, hence $\sim_{10}=\sim_{11}$. Otherwise, if $(P(\omega) / \text { Fin })^{+} \not \equiv$ $\left((P(\omega) / \text { Fin })^{+}\right)^{2}$, then for $U_{1}, U_{2} \subset \omega$ satisfying $U_{1} \sim_{10} U_{2}$ by Fact 1.1 (b) we have sq $\mathbb{P}\left(U_{1}\right) \equiv \mathrm{sq} \mathbb{P}\left(U_{2}\right)$ so, by the assumption and (20), sq $\mathbb{P}\left(U_{1}\right) \cong \mathrm{sq} \mathbb{P}\left(U_{2}\right)$. Thus $\sim_{10} \subset \sim_{8}$ and, hence $\sim_{10}=\sim_{8}=\sim_{6}$.

By Fact 3.4 b), CH implies that $(P(\omega) / \text { Fin })^{+} \equiv\left((P(\omega) / \text { Fin })^{+}\right)^{2}$. But, by a result of Shelah and Spinas [14], in the Mathias model these two posets have different distributivity numbers and, hence, they are not forcing equivalent. 


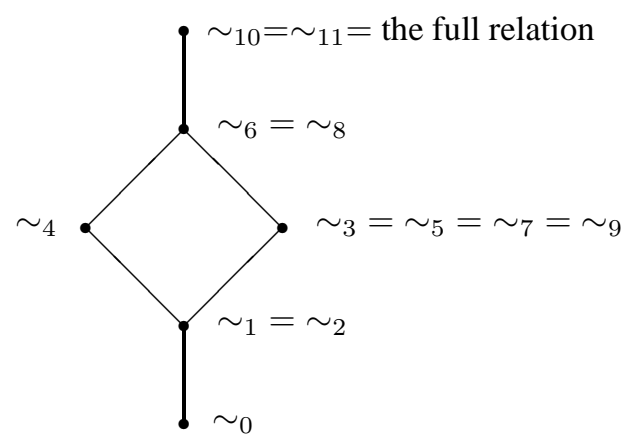

Figure 5: The similarities on $\operatorname{Mod}_{\langle R\rangle}(\omega)$ if $(P(\omega) / \text { Fin })^{+} \equiv\left((P(\omega) / \text { Fin })^{+}\right)^{2}$

\subsection{Infinite non-unary structures}

For infinite structures of non-unary languages the diagram from Figure 2 does not collapse at all. Namely the main result of this subsection is the following theorem.

Theorem 3.7 If $L$ is a non-unary relational language and $\kappa$ an infinite cardinal, then in the diagram from Figure 2 describing the similarities $\sim_{k}$ on the set $\operatorname{Mod}_{L}(\kappa)$ all the implications $a$-o are proper and there are no new implications (except the ones following from transitivity). Consequently, the same holds for the diagram from Figure $\square$ related to the class of all relational structures.

Theorem 3.7 will be proved in two steps. First we will prove the statement for the class $\operatorname{Mod}_{L_{b}}(\omega)$ of countable binary structures (where $L_{b}=\langle R\rangle$ and $\operatorname{ar}(R)=2$ ) and then, roughly speaking, make a correspondence between the classes $\operatorname{Mod}_{L_{b}}(\omega)$ and $\operatorname{Mod}_{L}(\kappa)$ preserving all the similarities $\sim_{k}$ and their negations.

\subsubsection{Proof of Theorem 3.7 for the class of countable binary structures}

First, giving examples (i.e. constructing pairs of structures), we show that for $L=$ $L_{b}$ and $|X|=\omega$, in the diagram from Figure 2 all the implications $a-o$ are proper. We will use the following auxiliary claim.

Lemma 3.8 If $\mathbb{P}=\left\langle P, \leq_{P}\right\rangle$ and $\mathbb{Q}=\left\langle Q, \leq_{Q}\right\rangle$ are partial orders and $f: P \rightarrow Q$ a surjection such that for each $p_{1}, p_{2} \in P$ we have

(i) $p_{1} \leq_{P} p_{2} \Rightarrow f\left(p_{1}\right) \leq_{Q}^{*} f\left(p_{2}\right)$,

(ii) $p_{1} \perp_{P} p_{2} \Rightarrow f\left(p_{1}\right) \perp_{Q} f\left(p_{2}\right)$,

then $\mathrm{sq} \mathbb{P} \cong \mathrm{sq} \mathbb{Q}$. 
Proof. First we prove that for each $p_{1}, p_{2} \in P$ we have

$$
p_{1} \leq_{P}^{*} p_{2} \Leftrightarrow f\left(p_{1}\right) \leq_{Q}^{*} f\left(p_{2}\right) .
$$

$(\Rightarrow)$ Assuming $p_{1} \leq_{P}^{*} p_{2}$ we have to prove that

$$
\forall q \leq_{Q} f\left(p_{1}\right) q \not \swarrow_{Q} f\left(p_{2}\right) .
$$

Let $q \leq_{Q} f\left(p_{1}\right)$. Since $f$ is onto, there is $p_{3} \in P$ such that $f\left(p_{3}\right)=q$. Thus $f\left(p_{3}\right) \leq_{Q} f\left(p_{1}\right)$ and, by (ii), there is $p_{4} \leq_{P} p_{3}, p_{1}$. So, since $p_{1} \leq_{P}^{*} p_{2}$ we have $p_{4} \not \chi_{P} p_{2}$ namely there is $p_{5} \leq_{P} p_{4}, p_{2}$. By (i) we have $f\left(p_{5}\right) \leq_{Q}^{*} f\left(p_{2}\right)$, which implies $f\left(p_{5}\right) \not \swarrow_{Q} f\left(p_{2}\right)$ and, hence, there is $q_{0} \leq_{Q} f\left(p_{5}\right), f\left(p_{2}\right)$. Since $p_{5} \leq_{P} p_{4} \leq_{P} p_{3}$, by (i) we have $f\left(p_{5}\right) \leq_{Q}^{*} f\left(p_{3}\right)=q$ and, hence, $q_{0} \leq_{Q}^{*} q$, which implies $q_{0} \not_{Q} q$, so there is $q^{\prime} \leq_{Q} q_{0}, q$. Now $q^{\prime} \leq_{Q} q, f\left(p_{2}\right)$ and (22) is proved.

$(\Leftarrow)$ Assuming (22) we prove that $p_{1} \leq_{P}^{*} p_{2}$. So, taking $p \leq_{P} p_{1}$ we show that $p \not \perp_{P} p_{2}$. By (i) we have $f(p) \leq_{Q}^{*} f\left(p_{1}\right)$ which implies that there is $q \leq_{Q}$ $f(p), f\left(p_{1}\right)$. By (22) we have $q \not \perp_{Q} f\left(p_{2}\right)$ and, hence, there is $q^{\prime} \leq_{Q} q, f\left(p_{2}\right)$. Now $q^{\prime} \leq_{Q} f(p), f\left(p_{2}\right)$ and, by (ii), $p \not \perp_{P} p_{2}$. Thus (21) is proved.

Now we show that $\left\langle P /=_{P}^{*}, \unlhd_{P}\right\rangle \cong_{F}\left\langle Q /=_{Q}^{*}, \unlhd_{Q}\right\rangle$, where $F([p])=[f(p)]$. By (21), for $p_{1}, p_{2} \in P$ we have $\left[p_{1}\right]=\left[p_{2}\right]$ iff $p_{1}={ }_{P}^{*} p_{2}$ iff $p_{1} \leq_{P}^{*} p_{2} \wedge p_{2} \leq_{P}^{*} p_{1}$ iff $f\left(p_{1}\right) \leq_{Q}^{*} f\left(p_{2}\right) \wedge f\left(p_{2}\right) \leq_{Q}^{*} f\left(p_{1}\right)$ iff $f\left(p_{1}\right)=_{Q}^{*} f\left(p_{2}\right)$ iff $\left[f\left(p_{1}\right)\right]=\left[f\left(p_{2}\right)\right]$ iff $F\left(\left[p_{1}\right]\right)=F\left(\left[p_{2}\right]\right)$ and $F$ is a well defined injection. Since $f$ is onto, for $q \in Q$ there is $p \in P$ such that $q=f(p)$. Thus $F([p])=[f(p)]=[q]$ and $F$ is onto.

By (21) again, $\left[p_{1}\right] \unlhd_{P}\left[p_{2}\right]$ iff $p_{1} \leq_{P}^{*} p_{2}$ iff $f\left(p_{1}\right) \leq_{Q}^{*} f\left(p_{2}\right)$ iff $\left[f\left(p_{1}\right)\right] \unlhd_{Q}$ $\left[f\left(p_{2}\right)\right]$ iff $F\left(\left[p_{1}\right]\right) \unlhd_{Q} F\left(\left[p_{2}\right]\right)$. Thus $F$ is an isomorphism.

Example 3.9 The implication $a$ can not be reversed. Let $\mathbb{X}=\langle\omega, \leq\rangle$ and $\mathbb{Y}=$ $\left\langle\omega, \leq_{f}\right\rangle$, where $f: \omega \rightarrow \omega$ is a bijection different from the identity and $\leq_{f}=$ $\{\langle f(m), f(n)\rangle: m \leq n\}$. Then $\mathbb{X} \cong \mathbb{Y}$ and $\mathbb{P}(\mathbb{X})=\mathbb{P}(\mathbb{Y})=[\omega]^{\omega}$, but $\mathbb{X} \neq \mathbb{Y}$.

Example 3.10 The implications $b$ and $f$ can not be reversed. Let

$\mathbb{X}=\langle\omega,\{\langle n, n+1\rangle: n \in \omega\} \cup\{\langle 2 n, 2 n\rangle: n \in \omega\}\rangle$ and

$\mathbb{Y}=\langle\omega,\{\langle n, n+1\rangle: n \in \omega\} \cup\{\langle 2 n+1,2 n+1\rangle: n \in \omega\}\rangle$.

Then $\mathbb{P}(\mathbb{X})=\mathbb{P}(\mathbb{Y})=\{[2 n, \infty): n \in \omega\}$ and $\mathbb{X} \rightleftarrows \mathbb{Y}$ but $\mathbb{X} \not \mathbb{Y}$.

Example 3.11 The implications $c, e$ and $g$ can not be reversed. Let us define $\mathbb{X}=\left\langle\omega, \omega^{2} \backslash\{\langle 0,0\rangle\}\right\rangle$ and $\mathbb{Y}=\left\langle\omega, \omega^{2} \backslash\{\langle 1,1\rangle\}\right\rangle$. Then $\mathbb{X} \cong \mathbb{Y}$ and $\mathbb{P}(\mathbb{X})=$ $\left\{A \in[\omega]^{\omega}: 0 \in A\right\} \cong \mathbb{P}(\mathbb{Y})=\left\{A \in[\omega]^{\omega}: 1 \in A\right\}$, but $\mathbb{P}(\mathbb{X}) \neq \mathbb{P}(\mathbb{Y})$.

Example 3.12 The implications $d, h, k$ and $n$ can not be reversed. Let $\mathbb{X}=\langle\omega, \leq\rangle$ and $\mathbb{Y}=\langle\omega, \omega \times \omega\rangle$. Then $\mathbb{P}(\mathbb{X})=\mathbb{P}(\mathbb{Y})=[\omega]^{\omega}$ and, hence, $\mathbb{P}(\mathbb{X}) \cong \mathbb{P}(\mathbb{Y})$, $\mathrm{sq} \mathbb{P}(\mathbb{X}) \cong \mathrm{sq} \mathbb{P}(\mathbb{Y})$ and $\mathbb{P}(\mathbb{X}) \equiv \mathbb{P}(\mathbb{Y})$, but $\mathbb{X} \not \subset \mathbb{Y}$. 
Example 3.13 The implications $i$ and $j$ can not be reversed. Let $\mathbb{X}=\left\langle(0,1)_{\mathbb{Q}}, \leq\right\rangle$ and $\mathbb{Y}=\left\langle(0,1]_{\mathbb{Q}}, \leq\right\rangle$ be suborders of the rational line, $\mathbb{Q}$. Then, clearly, $\mathbb{X} \rightleftarrows \mathbb{Y}$.

Since the elements of $\mathbb{P}(\mathbb{X})$ are dense linear orders without end points, each chain $€$ in the poset $\langle\mathbb{P}(\mathbb{X}), \subset\rangle$ has a supremum: $\bigcup €$. On the other hand, $€=$ $\left\{\left(0, \frac{1}{2}-\frac{1}{n}\right]_{\mathbb{Q}}: n \geq 3\right\}$ is a chain in the poset $\langle\mathbb{P}(\mathbb{Y}), \subset\rangle, \bigcup €=\left(0, \frac{1}{2}\right) \notin \mathbb{P}(\mathbb{Y})$ and the sets $\left(0, \frac{1}{2}\right)_{\mathbb{Q}} \cup\{q\}, q \in\left[\frac{1}{2}, 1\right]_{\mathbb{Q}}$, are upper bounds for $€$, but $€$ does not have a least upper bound. Thus the poset $\langle\mathbb{P}(\mathbb{Y}), \subset\rangle$ is not chain complete and, hence, $\mathbb{P}(\mathbb{X}) \neq \mathbb{P}(\mathbb{Y})$.

Using Lemma 3.8 we show that $\mathrm{sq} \mathbb{P}(\mathbb{X}) \cong \mathrm{sq} \mathbb{P}(\mathbb{Y})$. We remind the reader that a linear order $L$ is called scattered iff $\mathbb{Q} \nrightarrow L$. Let Scatt denote the set of scattered suborders of $\mathbb{Q}$. It is easy to see that for $A, B \in \mathbb{P}(\mathbb{X})$ we have $A \leq^{*} B \Leftrightarrow A \backslash B \in$ Scatt and $A \perp B \Leftrightarrow A \cap B \in$ Scatt (where $\leq *$ is the corresponding separative modification) and that the same holds for $A, B \in \mathbb{P}(\mathbb{Y})$. Clearly, if $A \in \mathbb{P}(\mathbb{Y})$, then $A \backslash\{\max A\} \subset(0,1)_{\mathbb{Q}}$ and it is a copy of $\mathbb{X}$, so, the function $f: \mathbb{P}(\mathbb{Y}) \rightarrow \mathbb{P}(\mathbb{X})$, given by $f(A)=A \backslash\{\max A\}$, is well defined and we show that it satisfies the assumptions of Lemma 3.8. First, if $C \in \mathbb{P}(\mathbb{X})$, then $C \subset(0,1)_{\mathbb{Q}}$ and, clearly, $C \cup\{1\} \in \mathbb{P}(\mathbb{Y})$ and $f(C \cup\{1\})=C$. Thus $f$ is a surjection. Let $A, B \in \mathbb{P}(\mathbb{Y})$. If $A \subset B$, then $f(A) \backslash f(B)=(A \backslash\{\max A\}) \backslash(B \backslash\{\max B\}) \subset\{\max B\} \in$ Scatt and, hence, $f(A) \leq^{*} f(B)$ so (i) is true. If $A \perp B$, that is $A \cap B \in$ Scatt, then, clearly, $f(A) \cap f(B) \in$ Scatt, thus $f(A) \perp f(B)$ and (ii) is true as well. By Lemma 3.8 we have sq $\mathbb{P}(\mathbb{X}) \cong \operatorname{sq} \mathbb{P}(\mathbb{Y})$.

Example 3.14 The implication $m$ can not be reversed. By Example 4.4 of [6], if $\mathbb{X}$ is the directed graph $\left\langle\omega_{2}, \rho\right\rangle$, where $\rho=\left\{\left\langle\varphi, \varphi^{\wedge} i\right\rangle: \varphi \in{ }^{<\omega_{2}} \wedge i \in 2\right\}$, then $\langle\mathbb{P}(\mathbb{X}), \subset\rangle=\mathrm{sq}\langle\mathbb{P}(\mathbb{X}), \subset\rangle \cong\left\langle<\omega_{2}, \supset\right\rangle$. Let $\mathbb{Y}$ be the directed graph $\left\langle{ }^{<\omega} 3, \sigma\right\rangle$, where $\sigma=\left\{\left\langle\varphi, \varphi^{\wedge} i\right\rangle: \varphi \in{ }^{<\omega} 3 \wedge i \in 3\right\}$, then in a similar way we show that $\langle\mathbb{P}(\mathbb{Y}), \subset\rangle=\mathrm{sq}\langle\mathbb{P}(\mathbb{Y}), \subset\rangle \cong\langle\langle\omega 3, \supset\rangle$. Clearly sq $\langle\mathbb{P}(\mathbb{X}), \subset\rangle ¥ \mathrm{sq}\langle\mathbb{P}(\mathbb{Y}), \subset\rangle$, but $\operatorname{rosq}\langle\mathbb{P}(\mathbb{X}), \subset\rangle \cong \operatorname{rosq}\langle\mathbb{P}(\mathbb{Y}), \subset\rangle \cong \operatorname{Borel} / \mathcal{M}$.

Example 3.15 The implication $l$ can not be reversed. Let $\mathbb{X}$ be the directed graph from Example 3.14 and let $\mathbb{Y}$ be the directed graph $\langle Y, \sigma\rangle$, where $Y \subset{ }^{<\omega} 2$ and $\sigma \subset Y \times Y$ are defined by

$$
\begin{gathered}
Y=\{\emptyset, 0,1\} \cup\left\{j j^{\wedge} \varphi: j \in 2 \wedge \varphi \in \epsilon^{<\omega} 2\right\}, \\
\sigma=\{\langle\emptyset, 0\rangle,\langle\emptyset, 1\rangle,\langle 0,00\rangle,\langle 1,11\rangle\} \cup\left\{\left\langle j j^{\wedge} \varphi, j j^{\wedge} \varphi^{\curvearrowright} k\right\rangle: j, k \in 2 \wedge \varphi \in^{<\omega_{2}} 2\right\} .
\end{gathered}
$$

It is easy to see that $\mathbb{X} \rightleftarrows \mathbb{Y}$ and

$$
\mathbb{P}(\mathbb{Y})=\{Y\} \cup\left\{A_{j j^{\wedge} \varphi}^{k l}: j, k, l \in 2 \wedge \varphi \in^{<\omega} 2\right\}
$$




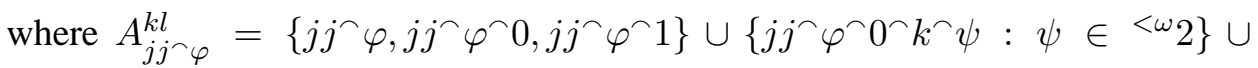
$\left\{j j^{\wedge} \varphi^{\wedge} 1^{\wedge} l^{\wedge} \psi: \psi \in{ }^{<\omega} 2\right\}$. By Example 3.14 the poset $\mathrm{sq}\langle\mathbb{P}(\mathbb{X}), \subset\rangle$ is isomorphic to the reversed binary tree. Thus, in order to prove that sq $\mathbb{P}(\mathbb{Y}) \approx \mathrm{sq} \mathbb{P}(\mathbb{X})$ we will show that $\left[A_{00}^{00}\right]$ and $\left[A_{00}^{01}\right]$ are incomparable but compatible elements of $\operatorname{sq} \mathbb{P}(\mathbb{Y})=\left\langle\mathbb{P}(\mathbb{Y}) /=^{*}, \unlhd\right\rangle$. So we have

$$
\begin{aligned}
& A_{00}^{00}=\{00,000,001\} \cup\left\{0000^{\curvearrowleft} \psi: \psi \in^{<\omega} 2\right\} \cup\left\{0010^{`} \psi: \psi \in^{<\omega} 2\right\}, \\
& A_{00}^{01}=\{00,000,001\} \cup\left\{0000^{\curvearrowleft} \psi: \psi \in^{<\omega} 2\right\} \cup\left\{0011^{\frown} \psi: \psi \in^{<\omega} 2\right\} .
\end{aligned}
$$

Clearly $\left\{0000^{\wedge} \psi: \psi \in{ }^{<\omega} 2\right\}$ is a copy of $\mathbb{X}$ and, hence, contains a copy of $\mathbb{Y}$, say $B$. Since $B \subset A_{00}^{00}, A_{00}^{01}$ we have $B \leq^{*} A_{00}^{00}, A_{00}^{01}$ and $[B] \unlhd\left[A_{00}^{00}\right],\left[A_{00}^{01}\right]$ thus $\left[A_{00}^{00}\right]$ and $\left[A_{00}^{01}\right]$ are compatible elements of sq $\mathbb{P}(\mathbb{Y})$.

In order to prove that $\left[A_{00}^{00}\right] \unlhd\left[A_{00}^{01}\right]$ we need $C \in \mathbb{P}(\mathbb{Y})$ such that $C \subset A_{00}^{00}$ and $D \not \subset C \cap A_{00}^{01}$, for all $D \in \mathbb{P}(\mathbb{Y})$. Now $\left\{0010^{\wedge} \psi: \psi \in \epsilon^{<\omega} 2\right\} \subset A_{00}^{00}$ is a copy of $\mathbb{X}$ and, hence, contains a copy of $\mathbb{Y}$, say $C$. Since $\left\{0010^{\wedge} \psi: \psi \in \epsilon^{<\omega} 2\right\} \cap A_{00}^{01}=\emptyset$, we have $C \cap A_{00}^{01}=\emptyset$ and we are done. Thus $\left[A_{00}^{00}\right] \unlhd\left[A_{00}^{01}\right]$ and, similarly, $\left[A_{00}^{01}\right] \unlhd\left[A_{00}^{00}\right]$.

Thus in Figure 2 for $\operatorname{Mod}_{L_{b}}(\omega)$ all the implications $a-o$ are proper and we show that there are no new implications except the ones following from transitivity. So it remains to be shown that the eight pairs which are incomparable in the Hasse diagram in Figure 2 are really incomparable. We will use the following elementary fact: if $\mathbb{P}=\langle P, \leq\rangle$ is a partial order and $p, q, r \in P$, then

$$
r=p \wedge q \text { and } r<p \text { and } r<q \Rightarrow p \| q .
$$

In fact our poset of similarities is a suborder of the lattice $\left\langle E Q\left(\operatorname{Int}_{L_{b}}(\omega)\right), \subset\right\rangle$ of equivalence relations on the set $\operatorname{Int}_{L_{b}}(\omega)$, where for $\sim, \sim^{\prime} \in E Q\left(\operatorname{Int}_{L_{b}}(\omega)\right)$ we have $\sim \wedge \sim^{\prime}=\sim \cap \sim^{\prime}$ and $\sim \vee \sim^{\prime}=\operatorname{trcl}\left(\sim \cup \sim^{\prime}\right)$ and $\sim \subset \sim^{\prime}$ iff the $\sim$-partition is a refinement of the $\sim^{\prime}$-partition of $\operatorname{Int}_{L_{b}}(\omega)$. Now, since by our definition we have $\sim_{1}=\sim_{2} \cap \sim_{3}$, by (23) we obtain $\sim_{2} \| \sim_{3}$ and similarly for the other seven pairs.

\subsubsection{Proof of Theorem 3.7}

The following concepts and facts will be used in our proof. Let $L_{b}=\langle R\rangle$, where $\operatorname{ar}(R)=2$. If $\mathbb{X}=\langle X, \rho\rangle$ is an $L_{b}$-structure, then the transitive closure $\rho_{\text {rst }}$ of the relation $\rho_{r s}=\Delta_{X} \cup \rho \cup \rho^{-1}$ (given by $x \rho_{r s t} y$ iff there are $n \in \mathbb{N}$ and $z_{0}=x, z_{1}, \ldots, z_{n}=y$ such that $z_{i} \rho_{r s} z_{i+1}$, for each $\left.i<n\right)$ is the minimal equivalence relation on $X$ containing $\rho$. The corresponding equivalence classes are called the components of $\mathbb{X}$ and the structure $\mathbb{X}$ is called connected iff $\left|X / \rho_{\text {rst }}\right|=1$. 
The complement of the structure $\mathbb{X},\langle X,(X \times X) \backslash \rho\rangle$ will be denoted by $\mathbb{X}^{c}$; its reflexification, $\left\langle X, \rho \cup \Delta_{X}\right\rangle$, by $\mathbb{X}_{r e}$; and its irreflexification, $\left\langle X, \rho \backslash \Delta_{X}\right\rangle$, by $\mathbb{X}_{i r}$.

If $\mathbb{X}_{i}=\left\langle X_{i}, \rho_{i}\right\rangle, i \in I$, are connected $L_{b}$-structures and $X_{i} \cap X_{j}=\emptyset$, for different $i, j \in I$, then the structure $\bigcup_{i \in I} \mathbb{X}_{i}=\left\langle\bigcup_{i \in I} X_{i}, \bigcup_{i \in I} \rho_{i}\right\rangle$ is the disjoint union of the structures $\mathbb{X}_{i}, i \in I$, and the structures $\mathbb{X}_{i}, i \in I$, are its components.

Fact 3.16 ([6]) If $\mathbb{X}$ is an $L_{b}$-structure, then at least one of the structures $\mathbb{X}$ and $\mathbb{X}^{c}$ is connected.

Fact 3.17 ([6]) Let $\mathbb{X}_{i}=\left\langle X_{i}, \rho_{i}\right\rangle, i \in I$, and $\mathbb{Y}_{j}=\left\langle Y_{j}, \sigma_{j}\right\rangle, j \in J$, be families of disjoint connected binary structures. Then $F: \bigcup_{i \in I} \mathbb{X}_{i} \hookrightarrow \bigcup_{j \in J} \mathbb{Y}_{j}$ iff there are $f: I \rightarrow J$ and $g_{i}: \mathbb{X}_{i} \hookrightarrow \mathbb{Y}_{f(i)}, i \in I$, such that $F=\bigcup_{i \in I} g_{i}$ and $\left\langle g_{i}(x), g_{i^{\prime}}\left(x^{\prime}\right)\right\rangle \notin \sigma_{r s}$, whenever $i \neq i^{\prime}, x \in X_{i}$ and $x^{\prime} \in X_{i^{\prime}}$.

Fact 3.18 Let $\mathbb{X}$ be a binary structure. Then

(a) $\operatorname{Emb}(\mathbb{X})=\operatorname{Emb}\left(\mathbb{X}^{c}\right)$ and $\mathbb{P}(\mathbb{X})=\mathbb{P}\left(\mathbb{X}^{c}\right)$;

(b) If $\mathbb{X}$ is irreflexive, then $\operatorname{Emb}(\mathbb{X})=\operatorname{Emb}\left(\mathbb{X}_{r e}\right)$ and $\mathbb{P}(\mathbb{X})=\mathbb{P}\left(\mathbb{X}_{r e}\right)$;

(c) If $\mathbb{X}$ is reflexive, then $\operatorname{Emb}(\mathbb{X})=\operatorname{Emb}\left(\mathbb{X}_{i r}\right)$ and $\mathbb{P}(\mathbb{X})=\mathbb{P}\left(\mathbb{X}_{i r}\right)$.

Theorem 3.19 (Vopěnka, Pultr, Hedrlín [15]) On any set $X$ there is an irreflexive binary relation $\rho$ such that $\mathrm{id}_{X}$ is the only endomorphism of the structure $\langle X, \rho\rangle$.

For a cardinal $\lambda$ let $\operatorname{Int}_{L_{b}}^{*}(\lambda)=\left\{\rho \subset \lambda^{2}:\langle\lambda, \rho\rangle\right.$ is connected $\left.\wedge \rho \cap \Delta_{\lambda} \neq \emptyset\right\}$. Then $\operatorname{Int}_{L_{b}}^{*}(\lambda) \subset \operatorname{Int}_{L_{b}}(\lambda)$ and $\operatorname{Mod}_{L_{b}}^{*}(\lambda):=\left\{\langle\lambda, \rho\rangle: \rho \in \operatorname{Int}_{L_{b}}^{*}(\lambda)\right\} \subset \operatorname{Mod}_{L_{b}}(\lambda)$.

Theorem 3.20 Let $\kappa \geq \lambda \geq \omega$ be cardinals and $L=\left\langle R_{i}: i \in I\right\rangle$ a non-unary relational language. Then there is a mapping $\tau: \operatorname{Int}_{L_{b}}^{*}(\lambda) \rightarrow \operatorname{Int}_{L}(\kappa)$ such that

(a) $\mathbb{P}\left(\kappa, \tau_{\rho}\right) \cong \mathbb{P}(\lambda, \rho)$, for each $\rho \in \operatorname{Int}_{L_{b}}^{*}(\lambda)$;

(b) For each $\rho \in \operatorname{Int}_{L_{b}}(\lambda)$ there are $\rho^{\prime} \in \operatorname{Int}_{L_{b}}^{*}(\lambda)$ and $\tau \in \operatorname{Int}_{L}(\kappa)$ such that $\mathbb{P}\left(\lambda, \rho^{\prime}\right)=\mathbb{P}(\lambda, \rho) \cong \mathbb{P}(\kappa, \tau) ;$

(c) $\tau$ preserves all the relations $\sim_{k}$ from Figure 2 that is for each $k \leq 11$

$$
\forall \rho, \sigma \in \operatorname{Int}_{L_{b}}^{*}(\lambda)\left(\rho \sim_{k} \sigma \Leftrightarrow \tau_{\rho} \sim_{k} \tau_{\sigma}\right) .
$$

Proof. First suppose that $\lambda<\kappa$. Then $|\kappa \backslash \lambda|=\kappa$ and, by Theorem 3.19 we can fix an irreflexive binary relation $\theta \subset(\kappa \backslash \lambda)^{2}$ such that $\operatorname{Emb}(\kappa \backslash \lambda, \theta)=\left\{\operatorname{id}_{\kappa \backslash \lambda}\right\}$. By Theorem 3.16 and Facts 3.18 (a) and 3.18 (c) we can assume that the relation $\theta$ is connected and irreflexive. The language $L$ is not unary and we fix an $i_{0} \in I$ such that $n_{i_{0}} \geq 2$. Now, for $\rho \in \operatorname{Int}_{L_{b}}^{*}(\lambda)$ let the interpretation $\tau_{\rho}=\left\langle\tau_{i}^{\rho}: i \in I\right\rangle \in$ $\operatorname{Int}_{L}(\kappa)$ be defined by

$$
\tau_{i}^{\rho}=\left\{\begin{array}{cl}
(\rho \cup \theta) \times \kappa^{n_{i_{0}}-2} & \text { if } i=i_{0} \text { and } n_{i_{0}}>2 \\
(\rho \cup \theta) & \text { if } i=i_{0} \text { and } n_{i_{0}}=2 \\
\emptyset & \text { if } i \neq i_{0} .
\end{array}\right.
$$


For convenience, for $\rho, \sigma \in \operatorname{Int}_{L_{b}}^{*}(\lambda)$, instead of $\operatorname{Emb}\left(\left\langle\kappa, \tau_{\rho}\right\rangle,\left\langle\kappa, \tau_{\sigma}\right\rangle\right)$ (respectively, $\operatorname{Emb}(\langle\lambda, \rho\rangle,\langle\lambda, \sigma\rangle))$ we will write $\operatorname{Emb}\left(\tau_{\rho}, \tau_{\sigma}\right)(\operatorname{resp} . \operatorname{Emb}(\rho, \sigma))$.

Claim 3.21 For each $\rho, \sigma \in \operatorname{Int}_{L_{b}}^{*}(\lambda)$ we have

(i) $\operatorname{Emb}\left(\tau_{\rho}, \tau_{\sigma}\right)=\left\{f \cup \mathrm{id}_{\kappa \backslash \lambda}: f \in \operatorname{Emb}(\rho, \sigma)\right\}$;

(ii) $\operatorname{Iso}\left(\tau_{\rho}, \tau_{\sigma}\right)=\left\{f \cup \operatorname{id}_{\kappa \backslash \lambda}: f \in \operatorname{Iso}(\rho, \sigma)\right\}$;

(iii) $\mathbb{P}\left(\tau_{\rho}, \tau_{\sigma}\right)=\{C \cup(\kappa \backslash \lambda): C \in \mathbb{P}(\rho, \sigma)\}$;

(iv) $\operatorname{Emb}\left(\tau_{\rho}\right)=\left\{f \cup \operatorname{id}_{\kappa \backslash \lambda}: f \in \operatorname{Emb}(\rho)\right\}$;

(v) $\operatorname{Aut}\left(\tau_{\rho}\right)=\left\{f \cup \operatorname{id}_{\kappa \backslash \lambda}: f \in \operatorname{Aut}(\rho)\right\}$;

(vi) $\mathbb{P}\left(\tau_{\rho}\right)=\{C \cup(\kappa \backslash \lambda): C \in \mathbb{P}(\rho)\}$.

Proof. For convenience let $\pi_{\rho}:=\rho \cup \theta$, for $\rho \in \operatorname{Int}_{L_{b}}^{*}(\lambda)$. First we prove that

$$
\operatorname{Emb}\left(\left\langle\kappa, \pi_{\rho}\right\rangle,\left\langle\kappa, \pi_{\sigma}\right\rangle\right)=\left\{f \cup \operatorname{id}_{\kappa \backslash \lambda}: f \in \operatorname{Emb}(\rho, \sigma)\right\} .
$$

By the construction, $\left\langle\kappa, \pi_{\rho}\right\rangle=\langle\lambda, \rho\rangle \cup\langle\kappa \backslash \lambda, \theta\rangle$ and $\left\langle\kappa, \pi_{\sigma}\right\rangle=\langle\lambda, \sigma\rangle \cup\langle\kappa \backslash \lambda, \theta\rangle$ are partitions of the binary structures $\left\langle\kappa, \pi_{\rho}\right\rangle$ and $\left\langle\kappa, \pi_{\sigma}\right\rangle$ into their connectivity components. Since $\rho \cap \Delta_{\lambda} \neq \emptyset$ and $\theta$ is an irreflexive relation, we have $\langle\lambda, \rho\rangle \not \rightarrow$ $\langle\kappa \backslash \lambda, \theta\rangle$ and the inequality $\kappa>\lambda$ implies that $\langle\kappa \backslash \lambda, \theta\rangle \leftrightarrow\langle\lambda, \sigma\rangle$. So, by Theorem 3.17, $F \in \operatorname{Emb}\left(\left\langle\kappa, \pi_{\rho}\right\rangle,\left\langle\kappa, \pi_{\sigma}\right\rangle\right)$ iff $F\lceil\lambda \in \operatorname{Emb}(\langle\lambda, \rho\rangle,\langle\lambda, \sigma\rangle)$ and $F\left\lceil(\kappa \backslash \lambda) \in \operatorname{Emb}(\langle\kappa \backslash \lambda, \theta\rangle)=\left\{\operatorname{id}_{\kappa \backslash \lambda}\right\}\right.$ and (26) is proved.

Now we prove

$$
\operatorname{Emb}\left(\left\langle\kappa, \tau_{i_{0}}^{\rho}\right\rangle,\left\langle\kappa, \tau_{i_{0}}^{\sigma}\right\rangle\right)=\left\{f \cup \operatorname{id}_{\kappa \backslash \lambda}: f \in \operatorname{Emb}(\rho, \sigma)\right\} .
$$

If $F: \kappa \rightarrow \kappa$ is an injection, then $F \in \operatorname{Emb}\left(\left\langle\kappa, \tau_{i_{0}}^{\rho}\right\rangle,\left\langle\kappa, \tau_{i_{0}}^{\sigma}\right\rangle\right)$ iff for each $x_{1}, x_{2}, \ldots, x_{n_{i_{0}}} \in \kappa$

$\left\langle x_{1}, x_{2}, \ldots, x_{n_{i_{0}}}\right\rangle \in \pi_{\rho} \times \kappa^{n_{i_{0}}-2} \Leftrightarrow\left\langle F\left(x_{1}\right), F\left(x_{2}\right), \ldots, F\left(x_{n_{i_{0}}}\right)\right\rangle \in \pi_{\sigma} \times \kappa^{n_{i_{0}}-2}$

iff for each $x_{1}, x_{2} \in \kappa$ we have: $\left\langle x_{1}, x_{2}\right\rangle \in \pi_{\rho} \Leftrightarrow\left\langle F\left(x_{1}\right), F\left(x_{2}\right)\right\rangle \in \pi_{\sigma}$, iff $F \in \operatorname{Emb}\left(\left\langle\kappa, \pi_{\rho}\right\rangle,\left\langle\kappa, \pi_{\sigma}\right\rangle\right)$. Now (27) follows from (26).

(i) Clearly, $F \in \operatorname{Emb}\left(\tau_{\rho}, \tau_{\sigma}\right)$ iff $F \in \operatorname{Emb}\left(\left\langle\kappa, \tau_{i}^{\rho}\right\rangle,\left\langle\kappa, \tau_{i}^{\sigma}\right\rangle\right)$, for all $i \in I$. By (25) this holds iff $F \in \operatorname{Emb}\left(\left\langle\kappa, \tau_{i_{0}}^{\rho}\right\rangle,\left\langle\kappa, \tau_{i_{0}}^{\sigma}\right\rangle\right)$ and we apply (27).

(ii) If $f \in \operatorname{Emb}(\rho, \sigma)$ then $f \cup \operatorname{id}_{\kappa \backslash \lambda}$ is a surjection iff $f$ is a surjection iff $f \in \operatorname{Iso}(\rho, \sigma)$. Now we apply (i).

(iii) $A \in \mathbb{P}\left(\tau_{\rho}, \tau_{\sigma}\right)$ iff there is $F \in \operatorname{Emb}\left(\tau_{\rho}, \tau_{\sigma}\right)$ such that $A=F[\kappa]$ so, by (i), iff $A=f[\lambda] \cup(\kappa \backslash \lambda)$, for some $f \in \operatorname{Emb}(\rho, \sigma)$, iff $A=C \cup(\kappa \backslash \lambda)$, for some $C \in \mathbb{P}(\rho, \sigma)$.

Statements (iv), (v) and (vi) follow from (i), (ii) and (iii) respectively.

Now we prove the theorem. 
(a) By Claim 3.21 vi) we have $\mathbb{P}\left(\tau_{\rho}\right)=\{C \cup(\kappa \backslash \lambda): C \in \mathbb{P}(\rho)\}$ and it is easy to check that the mapping $F: \mathbb{P}(\rho) \rightarrow \mathbb{P}\left(\tau_{\rho}\right)$, defined by $F(C)=C \cup(\kappa \backslash \lambda)$, is an isomorphism of the posets $\langle\mathbb{P}(\rho), \subset\rangle$ and $\left\langle\mathbb{P}\left(\tau_{\rho}\right), \subset\right\rangle$.

(b) Let $\rho \in \operatorname{Int}_{L_{b}}(\lambda) \backslash \operatorname{Int}_{L_{b}}^{*}(\lambda)$. If $\rho$ is connected, then it is irreflexive, thus $\rho_{\text {re }} \in \operatorname{Int}_{L_{b}}^{*}(\lambda)$ and, by Fact $3.18(\mathrm{~b}), \mathbb{P}\left(\lambda, \rho_{\text {re }}\right)=\mathbb{P}(\lambda, \rho)$. Otherwise, by Theorem 3.16 the relation $\rho^{c}$ is connected and, by Fact 3.18 a $), \mathbb{P}\left(\lambda, \rho^{c}\right)=\mathbb{P}(\lambda, \rho)$. Now, if $\rho^{c} \cap \Delta_{\lambda} \neq \emptyset$, we have $\rho^{c} \in \operatorname{Int}_{L_{b}}^{*}(\lambda)$; otherwise $\left(\rho^{c}\right)_{r e} \in \operatorname{Int}_{L_{b}}^{*}(\lambda)$ and, by Fact 3.18 b), $\mathbb{P}\left(\lambda,\left(\rho^{c}\right)_{\text {re }}\right)=\mathbb{P}\left(\lambda, \rho^{c}\right)=\mathbb{P}(\lambda, \rho)$.

If $\rho \in \operatorname{Int}_{L_{b}}(\lambda)$ and $\rho^{\prime} \in \operatorname{Int}_{L_{b}}^{*}(\lambda)$, where $\mathbb{P}(\lambda, \rho)=\mathbb{P}\left(\lambda, \rho^{\prime}\right)$, then by (a) we have $\mathbb{P}\left(\lambda, \rho^{\prime}\right) \cong \mathbb{P}\left(\kappa, \tau_{\rho^{\prime}}\right)$, where $\tau_{\rho^{\prime}} \in \operatorname{Int}_{L}(\kappa)$. Thus $\mathbb{P}\left(\kappa, \tau_{\rho^{\prime}}\right) \cong \mathbb{P}(\lambda, \rho)$.

(c) It is sufficient to prove that the mapping $\tau: \operatorname{Int}_{L_{b}}^{*}(\lambda) \rightarrow \operatorname{Int}_{L}(\kappa)$ preserves the relations $\sim_{k}$, for $k \in\{0,3,4,6,8,9,10\}$. Let $\rho, \sigma \in \operatorname{Int}_{L_{b}}^{*}(\lambda)$.

$\sim_{0}: \rho=\sigma \Leftrightarrow \tau_{\rho}=\tau_{\sigma}$. By (25) we have: $\tau_{\rho}=\tau_{\sigma}$ iff $\tau_{i_{0}}^{\rho}=\tau_{i_{0}}^{\rho}$ iff $\rho \cup \theta=\sigma \cup \theta$ iff $\rho=\sigma$.

$\sim_{3}: \rho \cong \sigma \Leftrightarrow \tau_{\rho} \cong \tau_{\sigma}$. If $\rho \cong \sigma$ and $f \in \operatorname{Iso}(\rho, \sigma)$, then, by Claim 3.21(ii), $f \cup \operatorname{id}_{\kappa \backslash \lambda} \in \operatorname{Iso}\left(\tau_{\rho}, \tau_{\sigma}\right)$ and, hence, $\tau_{\rho} \cong \tau_{\sigma}$. Conversely, if $\tau_{\rho} \cong \tau_{\sigma}$ and $F \in$ Iso $\left(\tau_{\rho}, \tau_{\sigma}\right)$, then, by Claim 3.21 (ii), $F \uparrow \lambda \in \operatorname{Iso}(\rho, \sigma)$ and, hence, $\rho \cong \sigma$.

$\sim_{9}: \rho \rightleftarrows \sigma \Leftrightarrow \tau_{\rho} \rightleftarrows \tau_{\sigma}$. If $\rho \hookrightarrow \sigma$ and $f \in \operatorname{Emb}(\rho, \sigma)$, then, by Claim 3.21 (i), $f \cup \operatorname{id}_{\kappa \backslash \lambda} \in \operatorname{Emb}\left(\tau_{\rho}, \tau_{\sigma}\right)$ and, hence, $\tau_{\rho} \hookrightarrow \tau_{\sigma}$. Thus $\rho \rightleftarrows \sigma$ implies $\tau_{\rho} \rightleftarrows \tau_{\sigma}$. Conversely, if $\tau_{\rho} \hookrightarrow \tau_{\sigma}$ and $F \in \operatorname{Emb}\left(\tau_{\rho}, \tau_{\sigma}\right)$, then, by Claim 3.21 (i), $F\left\lceil\lambda \in \operatorname{Emb}(\rho, \sigma)\right.$ and, hence, $\rho \hookrightarrow \sigma$. So $\tau_{\rho} \rightleftarrows \tau_{\sigma}$ implies $\rho \rightleftarrows \sigma$.

$\sim_{4}: \mathbb{P}(\rho)=\mathbb{P}(\sigma) \Leftrightarrow \mathbb{P}\left(\tau_{\rho}\right)=\mathbb{P}\left(\tau_{\sigma}\right)$. This follows from Claim 3.21(vi).

$\sim_{6}: \mathbb{P}(\rho) \cong \mathbb{P}(\sigma) \Leftrightarrow \mathbb{P}\left(\tau_{\rho}\right) \cong \mathbb{P}\left(\tau_{\sigma}\right)$. This is true since by (a) we have

$$
\mathbb{P}(\rho) \cong \mathbb{P}\left(\tau_{\rho}\right) \text { and } \mathbb{P}(\sigma) \cong \mathbb{P}\left(\tau_{\sigma}\right)
$$

$\sim_{8}: \mathrm{sq} \mathbb{P}(\rho) \cong \mathrm{sq} \mathbb{P}(\sigma) \Leftrightarrow \mathrm{sq} \mathbb{P}\left(\tau_{\rho}\right) \cong \mathrm{sq} \mathbb{P}\left(\tau_{\sigma}\right)$. This is true since by (28) and Fact 1.1 a) we have sq $\mathbb{P}(\rho) \cong \operatorname{sq} \mathbb{P}\left(\tau_{\rho}\right)$ and $\operatorname{sq} \mathbb{P}(\sigma) \cong \operatorname{sq} \mathbb{P}\left(\tau_{\sigma}\right)$.

$\sim_{10}$ : rosq $\mathbb{P}(\rho) \cong \operatorname{rosq} \mathbb{P}(\sigma) \Leftrightarrow \operatorname{rosq} \mathbb{P}\left(\tau_{\rho}\right) \cong \operatorname{rosq} \mathbb{P}\left(\tau_{\sigma}\right)$. By (28) and Fact 1.1 (a) we have rosq $\mathbb{P}(\rho) \cong \operatorname{rosq} \mathbb{P}\left(\tau_{\rho}\right)$ and rosq $\mathbb{P}(\sigma) \cong \operatorname{rosq} \mathbb{P}\left(\tau_{\sigma}\right)$.

So, the theorem is proved for $\lambda<\kappa$. If $\lambda=\kappa$, then we define $\tau_{i_{0}}^{\rho}:=\rho \times \kappa^{n_{i_{0}}-2}$ and continue in the same way.

Finally we prove Theorem 3.7 In Subsection 3.2.1 it is shown that all the implications $a-o$ in Figure 2 for the class $\operatorname{Mod}_{L_{b}}(\omega)$ are proper. For example, concerning the implication $a$, in Example 3.9 we have constructed $\rho, \sigma \in \operatorname{Int}_{L_{b}}^{*}(\omega)$ such that $\rho \sim_{1} \sigma$ but $\rho \chi_{0} \sigma$. By Theorem 3.20 (c) we have $\tau_{\rho} \sim_{1} \tau_{\sigma}$ and $\tau_{\rho} \chi_{0} \tau_{\sigma}$, which implies that in Figure 2 for the class $\operatorname{Mod}_{L}(\kappa)$ the implication $a$ is proper as well. The reader will notice that the structures constructed in Examples 3.9-3.13 belong to $\operatorname{Int}_{L_{b}}^{*}(\omega)$ and that the structures constructed in Examples 3.14 and 3.15 are irreflexive. But their refexifications are in $\operatorname{Int}_{L_{b}}^{*}(\omega)$. Thus all the implications 
$a$ - $o$ in Figure 2 for the class $\operatorname{Mod}_{L}(\kappa)$ are proper and using the same argument as in Subsection 3.2.1 we conclude that there are no additional implications in the diagram describing the hierarchy of the considered similarities on the class $\operatorname{Mod}_{L}(\kappa)$.

\section{References}

[1] B. Balcar, P. Vopěnka, On systems of almost disjoint sets, Bull. Acad. Polon. Sci. Sér. Sci. Math. Astronom. Phys. 20 (1972) 421-424.

[2] B. Balcar, P. Simon, Disjoint refinement, in: J. D. Monk and R. Bonnet (Eds.), Handbook of Boolean algebras, Vol. 2, 333-388, Elsevier Science Publishers B.V., Amsterdam, 1989.

[3] T. Jech, Set Theory, 2nd corr. Edition, Springer, Berlin, 1997.

[4] S. Koppelberg, General Theory of Boolean Algebras, in: J. D. Monk and R. Bonnet (Eds.), Handbook of Boolean Algebras, Part I, Elsevier Science Publishers B.V., Amsterdam, 1989.

[5] K. Kunen, Set Theory, An Introduction to Independence Proofs, North-Holland, Amsterdam, 1980.

[6] M. S. Kurilić, From $A_{1}$ to $D_{5}$ : Towards a forcing-related classification of relational structures, J. Symbolic Logic 79,1 (2014) 279-295.

[7] M. S. Kurilić, Maximally embeddable components, Arch. Math. Logic 52,7 (2013) 793-808.

[8] M. S. Kurilić, Forcing with copies of countable ordinals, Proc. Amer. Math. Soc. (in print).

[9] M. S. Kurilić, Posets of copies of countable scattered linear orders, Ann. Pure Appl. Logic 165 (2014) 895-912.

[10] M. S. Kurilić, Isomorphic and strongly connected components, Arch. Math. Logic (in print) DOI10.1007/s00153-014-0399-2.

[11] M. S. Kurilić, S. Todorčević, Forcing by non-scattered sets, Ann. Pure Appl. Logic 163 (2012) 1299-1308.

[12] M. S. Kurilić, S. Todorčević, Copies of the random graph, (to appear).

[13] M. S. Kurilić, S. Todorčević, Copies of the random graph: the 2-localization, (to appear).

[14] S. Shelah, O. Spinas, The distributivity numbers of $P(\omega) /$ fin and its square, Trans. Amer. Math. Soc. 352,5 (2000) 2023-2047.

[15] P. Vopěnka, A. Pultr, Z. Hedrlín, A rigid relation exists on any set, Comment. Math. Univ. Carolinae 6 (1965) 149-155. 